\title{
THE ANNUAL MEETING IN ST. LOUIS
}

The fifty-ninth Annual Meeting of the American Mathematical Society was held at Washington University, St. Louis, Missouri, Saturday through Monday, December 27-29, 1952, in conjunction with meetings of the American Association for the Advancement of Science, the Association for Symbolic Logic, and the Mathematical Association of America.

Almost 500 people registered for the meetings, among whom were the following 339 members of the Society:

V. W. Adkisson, L. W. Akers, A. A. Albert, E. S. Allen, C. B. Allendoerfer, R. D. Anderson, B. M. Armstrong, Nachman Aronszajn, M. C. Ayer, W. G. Bade, B. J. Ball, I. A. Barnett, Iacopo Barsotti, R. G. Bartle, M. A. Basoco, P. T. Bateman, J. D. Baum, E. G. Begle, Stefan Bergman, R. H. Bing, A. H. Black, R. L. Blair, H. D. Block, H. W. Bode, Raoul Bott, Joseph Bram, R. W. Brink, A. R. Brown, R. H. Bruck, H. D. Brunk, P. B. Burcham, Sister M. Leonarda Burke, R. S. Burington, Jewell H. Bushey, R. K. Butz, S. S. Cairns, Eugenio Calabi, J. M. Calloway, H. H. Campaigne, K. H. Carlson, Maria Castellani, Lamberto Cesari, M. N. Chase, J. O. Chellevold, Y. W. Chen, R. V. Churchill, D. E. Coffey, Harvey Cohn, L. A. Colquitt, Damian Connelly, P. F. Conrad, Sister Rose Margaret Cook, Byron Cosby, Jr., W. A. Couch, N. A. Court, C. H. Cunkle, J. C. Currie, C. W. Curtis, J. M. Danskin, Robert Davies, W. E. Deskins, A. H. Diamond, J. L. Doob, H. P. Doole, Jim Douglas, T. L. Downs, W. C. Doyle, Nelson Dunford, J. P. Dunnett, W. L. Duren, Jr., William H. Durfee, S. E. Dyer, J. M. Earl, J. C. Eaves, Samuel Eilenberg, R. E. Ekstrom, J. D. Elder, H. M. Elliott, J. G. Elliott, D. O. Ellis, Bernard Epstein, D. H. Erkiletian, M. E. Estill, H. J. Ettlinger, G. M. Ewing, W. H. Fagerstrom, F. D. Faulkner, William Feller, W. T. Fishback, D. A. Flanders, Harley Flanders, W. C. Foreman, G. E. Forsythe, J. S. Frame, R. E. Fullerton, H. M. Gehman, J. J. Gergen, F. J. Gerst, Murray Gerstenhaber, Leonard Gillman, A. M. Gleason, Casper Goffman, H. E. Goheen, V. D. Gokhale, Michael Goldberg, S. H. Gould, R. F. Graesser, L. M. Graves, F. L. Griffin, J. S. Griffin, Jr., H. C. Griffith, Simon Gruenzweig, D. L. Guy, Felix Haas, D. T. Haimo, Franklin Haimo, Edwin Halfar, P. C. Hammer, J. R. Hanna, Frank Harary, H. G. Harp, Melvin Hausner, N. A. Haynes, I. L. Hebel, G. A. Hedlund, L. J. Heider, A. E. Heins, Henry Helson, Melvin Henriksen, I. N. Herstein, Fritz Herzog, Edwin Hewitt, T. H. Hildebrandt, T. W. Hildebrandt, Sister M. Laetitia Hill, J. J. L. Hinrichsen, F. E. Hohn, D. L. Holl, L. A. Hostinsky, J. A. Hratz, S. T. Hu, G. B. Huff, Ralph Hull, M. G. Humphreys, M. H. Ingraham, W. H. Ito, L. K. Jackson, H. G. Jacob, Nathan Jacobson, Fritz John, L. W. Johnson, B. W. Jones, F. B. Jones, P. S. Jones, W. C. Kalinowski, Irving Kaplansky, M. E. Kellar, J. L. Kelley, M. R. Kenner, J. R. F. Kent, Mother Marie Kernaghan, D. E. Kibbey, V. L. Klee, S. C. Kleene, George Klein, Erwin Kleinfeld, L. A. Knowler, J. C. Koken, L. A. Kokoris, G. L. Kreezer, R. J. Lambert, C. E. Langenhop, R. E. Langer, Paolo Lanzano, Leo Lapidus, H. D. Larsen, W. G. Leavitt, Joseph Lehner, R. A. Leibler, Walter Leighton, Benjamin Lepson, W. J. LeVeque, Sister Mary Teresine Lewis, H. D. Lipsich, Lee Lorch, G. G. Lorentz, L. L. Lowenstein, C. I. Lubin, H. T. McAdams, W. C. McDaniel, E. J. McShane, G. R. MacLane, Saunders MacLane, H. M. MacNeille, Szolem Mandelbrojt, C. G. Maple, Morris Marden, R. H. Marquis, J. M. Marr, E. C. Marth, W. S. Massey, Gaylord Merriman, E. A. Michael, R. J. Michel, R. R. Middlemiss, W. L. Miser, 
E. E. Moise, M. A. Moore, R. A. Moore, T. W. Moore, W. L. Moore, Marston Morse, T. A. Mossman, H. T. Muhly, G. G. Murray, Zeev Nehari, David Nelson, W. J. Nemerever, John von Neumann, Albert Newhouse, C. V. Newsom, T. A. Newton, C. O. Oakley, H. W. Oliver, W. R. Orton, Morris Ostrofsky, T. P. Palmer, O. O. Pardee, S. T. Parker, G. W. Patterson, L. E. Payne, W. H. Pell, H. P. Pettit, C. G. Pitner, J. C. Polley, G. B. Price, O. J. Ramler, J. F. Randolph, L. T. Ratner, L. M. Reagan, M. S. Rees, R. F. Reeves, Francis Regan, Haim Reingold, R. W. Rempfer, J. G. Renno, P. R. Rider, J. K. Riess, J. D. Riley, R. F. Rinehart, L. A. Ringenberg, R. A. Roberts, L. V. Robinson, L. D. Rodabaugh, Hartley Rogers, Jr., I. H. Rose, Alex Rosenberg, P. C. Rosenbloom, J. B. Rosser, W. C. Royster, L. A. Rubel, H. S. Ruse, Charles Saltzer, Hans Samelson, R. G. Sanger, H. M. Schaerf, R. D. Schafer, M. M. Schiffer, E. R. Schneckenburger, Lowell Schoenfeld, J. T. Schwartz, L. L. Scott, W. R. Scott, R. R. Seeber, Jr., George Seifert, J. B. Serrin, M. A. Seybold, Seymour Sherman, Harold Shniad, Marlow Sholander, Edward Silverman, Annette Sinclair, F. B. Sloss, D. M. Smiley, M. F. Smiley, James C. Smith, W. S. Snyder, E. J. Specht, H. D. Sprinkle, S. K. B. Stein, R. L. Sternberg, P. C. Suppes, Gabor Szegö, C. T. Taam, T. T. Tanimoto, Alfred Tarski, M. E. Taylor, H. P. Thielman, A. J. Tinkley, E. W. Titt, A. W. Tucker, A. R. Turquette, J. L. Ullman, W. R. Utz, Jr., B. O. Van Hook, G. B. Van Schaack, D. E. Van Tijn, H. E. Vaughan, D. C. Velesz, D. F. Votaw, T. L. Wade, Jr., G. L. Walker, R. J. Walker, H. S. Wall, C. Y. Wang, H. C. Wang, J. A. Ward, L. E. Ward, Jr., A. M. Wedel, J. V. Wehausen, L. M. Weiner, B. A. Welch, F. J. Weyl, George Whaples, P. M. Whitman, G. T. Whyburn, L. S. Whyburn, R. L. Wilder, A. B. Willcox, W. L. Williams, R. A. Willoughby, R. H. Wilson, Jr., G. N. Wollan, Oswald Wyler, C. T. Yang, Ti Yen, L. C. Young, J. W. T. Youngs.

The Josiah Willard Gibbs Lecture, on Topology and geometrical analysis, was presented by Professor Marston Morse of the Institute for Advanced Study on Saturday evening. President Detlev Bronk of Johns Hopkins University, president of the National Academy of Sciences, presided.

Professor John von Neumann of the Institute for Advanced Study gave his Retiring Presidential Address, A logical theory of automata, on Sunday afternoon. Professor S. C. Kleene presided.

At a joint session of Section A and the Society at 3:30 P.M., Monday, Professor R. L. Wilder of the University of Michigan gave an address, Origin and growth of mathematical concepts, as a retiring Vice President of the American Association for the Advancement of Science. Professor L. M. Graves presided.

Professor A. M. Gleason of Harvard University gave an address, Natural co-ordinate systems, on Saturday afternoon, on invitation of the Committee to Select Hour Speakers for Annual and Summer Meetings. Professor Nelson Dunford presided. Professor Gleason's paper, which dealt with Hilbert's fifth problem, was awarded the Newcomb Cleveland Thousand Dollar Prize Award for the outstanding paper at the meeting of the American Association for the Advancement of Science.

The Registration Headquarters for this meeting were in the 
Women's Building and adjacent to various lounges which were available at all times. This feature together with tea served in the afternoons contributed to the success of the meeting.

More formal entertainment was provided by an Opera Workshop Performance on Sunday evening and a Sightseeing Tour of Saint Louis on Monday.

A dinner for members of the mathematical organizations and their guests was held on Monday evening, December 29, in the Women's Building. Professor T. L. Downs was the toastmaster. The mathematical organizations were welcomed by Leslie J. Buchan, ViceChancellor of Washington University. Professor G. T. Whyburn, President Elect of the American Mathematical Society, Professor L. M. Graves, Vice President and Chairman of Section A of the American Association for the Advancement of Science, Professor C. B. Allendoerfer, Editor of the Mathematical Association of America, and Professor J. B. Rosser, President of the Association for Symbolic Logic, spoke for their respective organizations. A resolution of appreciation and thanks was offered by Professor J. S. Frame to Washington University, the Committee on Arrangements, and all who had contributed to the success of the meeting. The resolution was adopted by a rising vote.

The annual Business Meeting of the Society was held on Monday afternoon, December 29, 1952. The Secretary reported on the affairs of the Society.

At the annual election, in which 820 votes were cast, the following officers were elected.

Vice President, Dr. D. H. Lehmer.

Secretary, Professor E. G. Begle.

Treasurer, Dean A. E. Meder, Jr.

Associate Secretary, Professor L. W. Cohen.

Member of the Editorial Committee of the Bulletin, Professor G. B. Price.

Members of the Editorial Committee of the Transactions and Memoirs, Professors J. L. Doob and L. V. Ahlfors.

Member of the Editorial Committee of the Proceedings, Professor A. C. Schaeffer.

Member of the Editorial Committee of the Colloquium Publications, Professor Deane Montgomery.

Member of the Editorial Committee of Mathematical Reviews, Professor Einar Hille.

Member of the Editorial Committee of Mathematical Surveys, Professor Max Shiffman.

Members of the Committee on Printing and Publishing, Professors 
M. R. Hestenes, J. R. Kline, and C. J. Rees.

Representative on the Board of Editors of the American Journal of Mathematics, Professor Samuel Eilenberg.

Members-at-large of the Council, Professors F. B. Jones, E. E. Moise, B. J. Pettis, R. M. Thrall, and G. W. Whitehead.

Members of the Board of Trustees, Professors B. P. Gill, J. R. Kline, and W. T. Martin.

The Council met on Sunday evening, December 28, 1952.

The Secretary announced the election of the following forty-two persons to ordinary membership in the Society.

Mr. Robert MacColl Adams, Research Mathematician, University of Texas;

Mr. Philip Nye Armstrong, Engineer, International Business Machines, Inc., Endicott, New York;

Mr. Clarence F. Barr, Head, Department of Mathematics, University of Wyoming;

Mr. Jose de Barros Neto, Assistant, University of São Paulo, São Paulo, Brazil;

Mrs. Barbara Beechler Blair (Mrs. R. L.), Assistant Instructor, State University of Iowa;

Mr. Robert L. Blair, Instructor, State University of Iowa;

Dr. Claude W. Burrill, University of Manchester;

Mr. Harold Davis, Engineer, University of California, Los Angeles, California;

Mr. Roy Bryan Deal, Jr., Instructor, University of Oklahoma;

Mr. Robert Diaz-Fernandez, University of California, Berkeley, California;

Mr. Victor John Doberly, Accessocraft Products Corporation, New Rochelle, New York;

Mr. Rudolf F. Drenick, Manager, Analytical Group, RCA Victor Division, Camden, New Jersey;

Mr. Cecil Eugene Duncan, Stanford University;

Mr. Frederick William Gehring, Instructor, Harvard University;

Mr. William Lewis Gordon, Duke University;

Mr. James Ray Hanna, Associate Professor, University of Wichita;

Mr. Walter Julius Huebner, Jr., St. Louis University;

Mrs. Mary E. Knight, Mathematician, White Sands Proving Ground, Las Cruces, New Mexico;

Mr. Alfred Baker Lehman, University of Florida;

Rev. Charles Joseph Lewis, Brown University;

Mr. John Howard Maecher, Teaching Fellow, University of Miami;

Mr. Arne Magnus, Instructor, University of Kansas;

Dr. Lucien Massé, Vice President, Weiss Geophysical Corporation of Canada, Calgary, Alberta, Canada;

Mr. Edward Joseph Murphy, Member of Technical Staff, Bell Telephone Laboratories, Summit, New Jersey;

Mr. James Pachares, Research Assistant, University of North Carolina;

Mr. Prom Panitchpakdi, University of Kansas;

Mr. Robert Lee John Penn, General Manager, Mathematics Research and Publishing Company, Austin, Texas;

Mr. Donald Jeanne Ritchie, Mathematician, Bendix Aviation Corporation Research Laboratories, Detroit 3, Michigan;

Miss Anne Elizabeth Scheerer, University of Pennsylvania;

Mr. Ben Moore Seelbinder, Part-time Instructor, University of North Carolina; 
Mr. Edwin Seymour Shapiro, Mathematician, U. S. Naval Radiological Defense Laboratory, San Francisco 22, California;

Dr. Frank L. Spitzer, New York, New York;

Mr. Raimond Aldrich Struble, Aerodynamicist, Douglas Aircraft Company, Santa Monica, California;

Professor Patrick Colonel Suppes, Stanford University;

Dr. Robert Flemming Tate, Lecturer, University of California, Berkeley, California;

Mr. Morris Tenenbaum, Cornell University;

Dr. Jose Pasquel Tola, Director, Instituto de Matematicas, Universidad Mayor de San Marcos, Lima, Peru;

Mr. Tung Tsang, Assistant Chemist, Minneapolis-Honeywell Regulator Company, Minneapolis, Minnesota, and Department of Chemical Engineering, University of Minnesota;

Mr. Bernard Weitzer, Mathematician, Republic Aviation Corporation, Farmingdale, Long Island, New York;

Dr. Robert Lewis Westhafer, Associate Professor, New Mexico College of Agriculture and Mechanic Arts, State College, New Mexico;

Mr. J. Hunter White, Jr., District Representative, International Business Machines Corporation, River Edge, New Jersey;

Miss Irma Marian Wyman, Research Associate, Willow Run Research Center, Ypsilanti, Michigan.

It was reported that the following thirteen persons had been elected to membership on nomination of institutional members as indicated:

Harvard University: Mr. Shreeram Shankar Abhyanuar, Mr. Edward Holton Bensley, Mr. Gerald Gustave Bilodeau, Mr. Alexander Blair, Miss Marian Boykan, Mr. Richard Robinson Goldberg, and Mr. Seth L. Warner.

Lehigh University: Mr. Chester B. Sensenig.

State University of Iowa: Mr. Wendall Don Lindstrom.

University of New Hampshire: Mr. Armand Raymond Lamontagne.

Tulane University of Louisiana: Mr. Charles Edward Capel, Mr. William Munroe Faucett, and Mr. John Sanders Griffin.

The Secretary announced that the following had been admitted to the Society in accordance with reciprocity agreements with various mathematical organizations: London Mathematical Society: Mr. David George Kendall, Princeton University, Professor Douglas Geoffrey Northcott, the University of Sheffield, Sheffield, England, and Professor Edward Maitland Wright, King's College, Aberdeen, Scotland; Schweizerische Mathematische Gesellschaft: Dr. Alfred Otto Huber, Institute for Fluid Dynamics and Applied Mathematics, University of Maryland; Unione Matematica Italiana: Professor Lorenzo Calabi, Boston College, Chesnut Hill, Massachusetts.

Mount Holyoke College, South Hadley, Massachusetts, and the University of Utah, Salt Lake City, Utah, were elected to institutional membership.

The Secretary is pleased to report at this time that the ordinary membership of the Society is now 4483, including 346 nominees of 
institutional members and 40 life members. The membership of the Society continues to show a slow, but steady, growth. There are also 124 institutional members. The total attendance at all meetings in 1952 was 1998; the number of papers read was 733; there were 13 hour addresses, 4 Colloquium Lectures, 1 Gibbs Lecture, 1 Retiring Presidential Address, and 15 papers at the Applied Mathematics Symposium. The number of members attending at least one meeting was 1348 .

The following appointments by the President were reported: as a committee to recommend policies for a separate Memoirs Editorial Committee: J. L. Doob, Chairman, R. P. Boas, and S. C. Kleene; as a committee to recommend to the Council the award of the Bôcher Memorial Prize: L. V. Ahlfors, Chairman, Philip Franklin, and D. C. Spencer; as a committee to nominate members for the Policy Committee: P. A. Smith, Chairman, G. A. Hedlund, and A. W. Tucker; as a committee on translations from Russian and other foreign languages: R. P. Boas and Irving Kaplansky for a term of three years beginning July 1, 1952, R. E. Bellman and Hans Samelson for a term of two years beginning July 1, 1952, J. L. Doob for a period of one year beginning July 1, 1952, and R. P. Boas as chairman for a period of one year beginning July 1, 1952; as an organizing committee for summer institutes: A. A. Albert, Chairman, and Salomon Bochner for a term of three years beginning July 1, 1952, H. P. Robertson and Hassler Whitney for a term of two years beginning July 1, 1952, and Nathan Jacobson and Oscar Zariski for a period of one year beginning July 1, 1952; as an arrangements committee for the Sixth Symposium in Applied Mathematics to be held at the Corona Laboratories of the National Bureau of Standards on August 26-28, 1953: D. H. Lehmer, Chairman, H. F. Bohnenblust, J. H. Curtiss, J. W. Green, George Pólya, and John Todd; as Editor of Volume VI of the Proceedings of the Symposium in Applied Mathematics: J. H. Curtiss; as tellers for the 1952 annual election: Zeev Nehari and G. B. Van Schaack; as a member of the Committee on Applied Mathematics (for a period of three years beginning January 1, 1953): Shizuo Kakutani (committee now consists of M. H. Martin, Chairman, R. V. Churchill, F. J. Murray, Eric Reissner, and Shizuo Kakutani); as a member of the Committee on Places of Meetings (for a period of three years beginning January 1, 1953): E. R. Lorch (committee now consists of Orrin Frink, Chairman, M. F. Smiley, and E. R. Lorch); as members of the Committee on Visiting Lectureships: Garrett Birkhoff for a period of two years beginning January 1, 1953, and Einar Hille for a period of three years beginning January 1, 1953 (committee now consists of R. P. Boas, Jr., Chairman, Garrett Birkhoff, and Einar 
Hille); as liaison officer to the Quarterly of Applied Mathematics for a term of three years beginning January 1, 1953: A. H. Taub; as a member of the Committee to Select Hour Speakers for Summer and Annual Meetings for the period 1953-1954: Oscar Zariski (committee now consists of E. G. Begle, Chairman, Tibor Radó, and Oscar Zariski); as a member of the Committee to Select Hour Speakers for Eastern Sectional Meetings for the period 1953-1954: A. M. Gleason (committee now consists of L. W. Cohen, Chairman, R. D. Schafer, and A. M. Gleason); as a member of the Committee to Select Hour Speakers for Southeastern Sectional Meetings for the period 19531954: J. J. Gergen (committee now consists of W. M. Whyburn, Chairman, Wallace Givens, and J. J. Gergen); as a member of the Committee to Select Hour Speakers for Western Sectional Meetings for the period 1953-1954: E. E. Moise (committee now consists of J. W. T. Youngs, Chairman, Marshall Hall, Jr., and E. E. Moise); as a member of the Committee to Select Hour Speakers for Far Western Sectional Meetings for the period 1953-1954: R. D. James (committee now consists of J. W. Green, Chairman, R. P. Dilworth, and R. D. James).

The Secretary reported that Professor J. A. Dieudonne had accepted an invitation to deliver an hour address at the April 1953 meeting in Chicago.

The Council approved the following places of sectional meetings in 1953: The Midwest November meeting at Northwestern University and the Far West November meeting at the California Institute of Technology.

The Secretary reported that the Policy Committee for Mathematics had voted to join the incorporators of a Scientific Manpower Commission. This Commission will gather and publicize information concerning present and future needs for scientific manpower.

The Secretary reported that a reciprocity agreement has been concluded with the Swedish Mathematical Society.

The Secretary reported that the invitation to hold the $1953 \mathrm{Sum}$ mer Meeting at Laval University in Quebec had been withdrawn because of difficulties in obtaining housing. The Council voted to accept an invitation from Queen's University and the Royal Military College to hold the Summer Meeting in Kingston, Ontario.

The Council approved the substitution of Professor R. P. Boas for Professor William Feller as a member of the Mathematical Reviews Editorial Board from February to August 1953.

The Bulletin Editorial Committee reported that the Journal used 718 pages in 1952, but that the increased size of reports of meetings and other material not under the control of the Editors of the Bul- 
letin had resulted in fewer pages of invited addresses. The Council voted to recommend to the Board of Trustees that a total of 625 pages be authorized for the 1953 Bulletin.

The Transactions and Memoirs Editorial Committee reported that the interval between receipt of a manuscript and publication is approximately nine months. The Council voted to recommend to the Trustees that 1100 pages be published in the Transactions for 1953.

The Mathematical Reviews Editorial Committee reported that 1014 pages had been published in 1952. The subscription list as of November 1952 was 2222.

The Proceedings Editorial Committee reported that the interval between receipt of a manuscript and publication had decreased to nine or ten months. The Council voted to recommend to the Board of Trustees that 1000 pages be authorized for the 1953 Proceedings. Professor R. C. Buck, E. A. Coddington, E. R. Kolchin, and E. E. Moise were reported as new assistant editors for the Proceedings.

The Colloquium Editorial Committee recommended that there be no Colloquium lectures in 1954, since the International Congress in Amsterdam will be held September 2-9, partially overlapping our summer meeting. The Council voted to approve this recommendation.

The Council elected Professor A. A. Albert as a representative of the Society on the Policy Committee for Mathematics for a fouryear term beginning January 1, 1953.

The Council voted to invite Professor Wassily Leontieff of Harvard University to deliver the Gibbs Lecture for 1953.

Professor A. A. Albert reported that the National Science Foundation had granted $\$ 20,000$ to the Society for a Summer Institute on Lie Groups and Lie Algebras. Professor Nathan Jacobson has been named Chairman of this Institute.

The Council voted to approve amendments to our by-laws which would: (1) empower the Council, under certain conditions, to speak in the name of the Society with respect to matters affecting the status, dignity, and effective position of mathematics or mathematicians; (2) require a referendum on such Council actions when requested by 200 or more members of the Society; (3) require a business meeting at the Summer as well as the Annual Meeting; (4) allow final action to be taken at a business meeting only when business is accepted by unanimous consent or notified to the full membership of the Society in the call for the meeting. These by-laws will be presented to the Society for approval at a business meeting at the time of the Summer Meeting.

Abstracts of the papers read follow. Presiding officers at the sessions 
for contributed papers were Dr. Seymour Sherman, Professor Saunders MacLane, Dr. A. H. Diamond, Professors G. B. Price, J. L. Kelley, Nathan Jacobson, D. L. Holl, A. A. Albert, E. E. Moise, Szolem Mandelbrojt, W. L. Duren, Jr., Ralph Hull, and Morris Marden.

Papers whose abstract numbers are followed by the letter " $t$ " were read by title. Paper number 159 was presented by Professor Herstein, 179 by Professor Stefan Bergman, 186 by Professor Schiffer, 193 by Professor Lorentz, 221 by Professor P. R. Rider, 238 by Mr. Minagawa, 241 by Professor Suppes, 252 by Professor Samelson, 255 by Mr. Sprinkle, and 258 by Dr. Harary.

Dr. Huber was introduced by Professor Alexander Weinstein, Mr. Griffin by Professor S. T. Hu, Professor Toralballa by Professor J. W. T. Youngs, Mr. Fettis by Professor P. R. Rider, Dr. Spitzer by Professor D. A. Darling, Dr. Jaeger by Professor S. S. Cairns, Dr. Bremermann by Professor Stefan Bergman.

\section{Algebra AND Theory of Numbers}

\section{6t. H. W. Becker: The Rignaux and Lebesgue transforms of Pythagorean tetrahedrons.}

Let the six edges of a Pythagorean tetrahedron be $u, v, x, y, z, t$, and the corresponding edges of tet. under transform $H$ be $t x, u v, u x, x z, t y, t u$; and under the Petrus transform of the first kind $P$, be $t^{2}-y^{2}=x^{2}+z^{2}, 2 u v, u^{2}-v^{2}=x^{2}-z^{2}, 2 x z, 2 t y, t^{2}$ $+y^{2}=u^{2}+v^{2}$, Dickson's History II, p. 446. Rignaux, L'Intermediaire des Math. vol. 25 (1918) p. 129, gave a transform $R$ on Petrus tets. under which (putting $T U+T X$ $+U X=\Delta$, and including the three edges $D \Delta$ unnecessary to his purposes) the edges $U, \cdots, T \rightarrow T^{2}-Y^{2}+\Delta, 2 V((T+U)(U+X))^{1 / 2}, U^{2}-V^{2}+\Delta, 2 Z((T+X)(U+X))^{1 / 2}$, $2 Y((T+U)(T+X))^{1 / 2}, T^{2}+Y^{2}+\Delta$. The transform $P$ also iterates on Petrus tets., where obviously $R \supset P$. Lebesgue, Dickson ibid., p. 637, showed that a certain quartic $=\square$ is a relative invariant under his transform $L$. Applying this to the tet. $u, \cdots, t=\left(\left(e^{2} g^{2}+f^{2} h^{2}\right)\left(e^{2} h^{2}+f^{2} g^{2}\right)\right)^{1 / 2}$, in the form $e, f, g, h \rightarrow e, f, e^{2} f^{2}\left(g^{4}-h^{4}\right)$, $2 t e f g h$, then $u, \cdots, t \rightarrow t^{2} y^{2}+u^{2} x^{2}, 2 t u v x, x^{4}-v^{2} y^{2}, 2 t u x y, 2 t u x z, t^{4}-v^{2} z^{2}$, and $L=R P$ $=P H$. Notwithstanding that $R P I \gg P^{2} I$, due to the rich possibilities of cancellation inherent in $H, R^{2}=H^{2}=I$, the identity, but $L^{2}=P^{2} \gg I$. A Pythagorean tet. also depends on $x=\left(\left(\epsilon^{2} \eta^{2}-\zeta^{2} \theta^{2}\right)\left(\epsilon^{2} \theta^{2}-\zeta^{2} \eta^{2}\right)\right)^{1 / 2}$. Under transform $\Lambda: \epsilon, \zeta, \eta, \theta \rightarrow \epsilon, \zeta, \epsilon^{2} \zeta^{2}\left(\eta^{4}-\theta^{4}\right)$, $2 x \epsilon \zeta \eta \theta ; u, \cdots, t \rightarrow 2 t u v z, t^{2} y^{2}+v^{2} z^{2}, 2 t v x z, 2 t v y z, z^{4}-u^{2} y^{2}, t^{4}-u^{2} x^{2}$. There are analogous transforms of the second kind: $G^{\prime}, P^{\prime}, R^{\prime}, L^{\prime}, \Lambda^{\prime}$, in a general way under permutations $(u v)(x z)$, with analogous inter-relations. But quite unexpectedly, $\Lambda=L^{\prime}, \Lambda^{\prime}=L$. (Received September 22, 1952.)

\section{7t. R. H. Bruck: Difference sets in a finite field.}

Let $F$ be a finite field of $P$ elements and let $H(m)$ be the multiplicative group of nonzero $m$ th powers in $F$, where $m$ divides $P-1$. The group $H(m)$ is a difference set in $F$ if the number of ordered pairs of elements $h, k$ of $H(m)$ such that $h-k=c$ is the same for each nonzero $c$ in $F$. A necessary condition is that $P \equiv 1+m \bmod m^{2}$; this assumed, the following conditions are necessary and sufficient that $H(m)$ be a 
difference set for the stated value of $m$ : (i) $m=2$ : none. (ii) $m=4: P=1+4 x^{2}$ for an integer $x$. (iii) $m=8: P=1+8 x^{2}=9+64 y^{2}$ for integers $x, y$. There are no difference sets $H(m)$ for $m=3,5,6$. Case (i) is trivial. In case (ii), $P$ must be prime; for $P$ prime the result was essentially proved by Gauss and reproved by Chowla (Proc. Nat. Acad. Sci. India, Sect. A, vol. 14 (1944) pp. 45-46). Case (iii) allows $P=9$, 73; other $P$ 's (if any) must exceed $10^{10}$. For indications of proof see the abstract on Gauss's method. (Received November 12, 1952.)

\section{R. H. Bruck: Gauss's method for the number of solutions of} certain equations in a finite field.

Let $g$ be a multiplicative generator of the finite field $F=G F(P)$ and let $H(m)$ be the multiplicative group of $m$ th powers, where $m$ divides $P-1$. The number of solutions in $F$ of $a x^{m}+\cdots+c z^{m}=d$, for any finite number of nonzero unknowns, $x, \cdots, z$, is expressible in terms of $(u, v)_{m}$, the number of ordered pairs of elements $h, k$ of $H(m)$ such that $g^{u} h+g^{v} k+1=0$. Gauss (for these and other references see A. Weil, Bull. Amer. Math. Soc. vol. 55 (1949) pp. 497-508) gave an elementary determination of $(u, v)_{m}$ for $P$ prime, $m=3$, 4. Later writers preferred to work with Gaussian or Jacobian sums. The present paper characterizes Gauss's method by giving a complete set of postulates for the number-theoretical functions $(u, v)_{m}$; the postulates concern $P$ but contain no reference to $G F(P)$. The group of mappings $(u, v)_{m} \rightarrow(r u, r v)_{m}, r$ prime to $m$, plays a central role, the function $(u, v)_{m}$ being expressible in terms of invariants of the group. The detailed determination of $(u, v)_{m}$ is given for $m=2,3,4,5,6,8$. (Received November 12, 1952.)

\section{J. M. Calloway: On the discriminant of arbitrary algebraic num-} ber fields.

An application of Minkowski's lemma on linear forms proves the result that the discriminant $d$ of every algebraic number field of degree $n>1$ is divisible by a prime number. An identity of C. L. Siegel (Nachr. Ges. Wiss. Göttingen (1922) pp. 17-24) gives this result for totally real fields. A generalization of Siegel's identity is obtained which shows that $|d|$ of an arbitrary algebraic number field of degree $n>1$ is greater than $(\pi / 3)^{2 r_{2}}\left(n=r_{1}+r_{2}\right)$. This is a special case of a second identity which also yields the Fourier expansion for the $(k+1)$ st Bernoulli polynomial. The identity is obtained in two ways. The first, a direct analogue of Siegel's method, consists of expanding a function in an $n$-fold Fourier series, multiplying both sides by a certain expression, and integrating. The second method consists of expanding a different function in an $n$-fold Fourier series. The second method while more elegant involves a more complicated argument as to the convergence of the Fourier series. (Received November 17, 1952.)

\section{0t. Leonard Carlitz: Congruences for the coefficients of the Weier-} strass $\wp$-function and related functions.

Let $\wp(x)=\wp\left(x ; g_{2}, g_{3}\right)$ denote the Weierstrass $\wp$-function, where $g_{2}, g_{3}$ are indeterminates. Put $f(x)=1 / \wp(x)=h^{2}(x)$; also let $h(x)=\sum_{1}^{\infty} A_{m} x^{m} / m !, f(x)$ $=\sum_{2}^{\infty} B_{m} x^{m} / m !, \wp(x)=x^{-2}+\sum_{2}^{\infty} C_{m} x^{m} / m !$, so that the $A_{m}, B_{m}, C_{m}$ are polynomials in $g_{2}, g_{3}$ with integral coefficients. Let $p$ be a fixed odd prime. The principal object of the paper is to prove congruences of the type $\sum_{s=0}^{r}(-1)^{r-s} C_{r, s} A_{p}^{r-s} B_{m+s(p-1)} \equiv 0$ $\left(\bmod p^{r}\right)$ for $m \geqq r \geqq 1$. For the $A_{m}$ we are unable to prove quite so much. (Received October 20, 1952.) 
151t. Leonard Carlitz: Kummer's congruences and the Schur derivative.

Let $p$ be a fixed prime and $\left\{a_{m}\right\}$ a sequence of rational numbers that are integral $(\bmod p)$. Define $\Delta a_{p^{m}}=\left(a_{p^{m+1}}-a_{p}^{p^{m}} a_{p^{m}}\right) / p^{m+1}, \Delta^{r} a_{p^{m}}=\left(\Delta^{r-1} a_{p^{m+1}}-a_{p}^{p^{m+m-1}} a_{p^{m}}\right) p^{m+1}$. The main result of the paper is that if $\sum_{s=1}^{r}(-1)^{r-s} C_{r, s} a_{p}^{r-s} a_{m+s(p-1)} \equiv 0\left(\bmod p^{r}\right)$ for $m \geqq r \geqq 1$, then $\Delta^{r} a_{p^{m}}, 1 \leqq r \leqq p-1$, are integral for all $m$. (Received October 18, 1952.)

\section{2t. Leonard Carlitz: Some theorems on Kummer's congruences.}

Let $\left\{a_{m}\right\}$ be a sequence of rational numbers that are integral $(\bmod p)$, where $p$ is a fixed prime. The relation $\sum_{s=0}^{r}(-1)^{r-s} C_{r, s} a_{m+s(p-1)} a_{p}^{r-s} \equiv 0\left(\bmod p^{r}\right)$ for all $m \geqq r$ $\geqq 1$ may be called Kummer's congruence for $\left\{a_{m}\right\}$. In the present paper it is proved for example that if $\left\{a_{m}\right\}$ and $\left\{b_{m}\right\}$ satisfy Kummer's congruence, then the same is true of $\left\{a_{m} b_{m}\right\}$; also if $a_{m}^{(k)}=m^{k} a_{m}$, then $a_{m}^{(k)}$ satisfies a congruence of a similar kind. If some additional hypotheses are introduced then one can assert that $\left\{c_{m}\right\}$ satisfies Kummer's congruence, where $c_{m}=\sum_{0}^{m} C_{m, s} a_{s} b_{m-s}$. (Received November 10,1952.)

153t. Eckford Cohen: Rings of arithmetic functions. II. Quadratic congruences.

Let $r$ be an arbitrary odd number greater than 1 , and let $a_{1}, \cdots, a_{s}$ be integers prime to $r$. The arithmetic part of this paper is concerned with the number of solutions $N=N_{s}(n, r)$ of the congruence $n \equiv a_{1} x_{1}^{2}+\cdots+a_{s} x_{s}^{2}(\bmod r)$. The author defines for this problem a finite "singular series" $\subseteq=\mathfrak{S}_{s}(n, r)$, analogous to the HardyRamanujan series for sums of squares in the nonmodular case. The function $\mathbb{S}$ is a divisor sum involving the familiar Ramanujan sum in case $s$ is even and a Gauss character sum in case $s$ is odd. Using orthogonality properties of these exponential sums, it is shown on the basis of induction that $N=\mathfrak{S}$. A direct proof of this fact is also given. The function $\subseteq$ is evaluated completely to give explicit arithmetic formulas for $N$, and several corollaries arising from special cases are obtained. Algebraic properties of arithmetic functions required in the above problem are discussed along the lines of Part I of this paper (Duke Math. J. vol. 19 (1952)). (Received December 29, 1952.)

154t. Eckford Cohen: On the number of solutions of cubic congruences.

Certain aspects of the method employed in the above paper with regard to quadratic congruences are used to determine the number of solutions $N=N\left(n, p^{\lambda}\right)$ of the cubic congruence $(\mathrm{I}), n \equiv a x^{3}+b y^{3}\left(\bmod p^{\lambda}\right)$, where $p$ is a prime $\equiv 1(\bmod 3)$, $(\lambda \geqq 1), a, b$ are integers prime to $p$, and $n$ is arbitrary. Denote by $t$ the largest integer $\leqq \lambda$ such that $n \equiv 0\left(\bmod p^{t}\right)$. Exact formulas for $N$ are obtained, with particularly simple results in case $t \equiv 1$ or $2(\bmod 3)$. From these formulas the following solvability criterion is deduced: If $p \neq 7$, then (I) is insolvable if and only if $t \neq 0(\bmod 3), t<\lambda$, and $a$ and $b$ have $a$ different cubic character $(\bmod p)$. The case $p=7$ is exceptional. A similar approach is applied to obtain the exact number of solutions $N^{\prime}=N^{\prime}\left(n, p^{\lambda}\right)$ of the congruence (II), $n \equiv a x^{3}+b y^{3}+c z^{3}\left(\bmod p^{\lambda}\right)$, where $p$ and $\lambda$ are defined as above and $(a b c, p)=1$. From this result is is verified that (II) is always solvable. Several corollaries, including approximations and recursion relations, are deduced as special cases of the results for $N$ and $N^{\prime}$. The author also obtains the number of primitive solutions 
of (I) and (II) $(x y z \neq 0(\bmod p))$ in the case of a prime modulus $p$. (Received December $29,1952$.

\section{5t. Harvey Cohn: Sign characterization of continued fractions.}

Consider triples of vectors of a two-dimensional plane lattice, $V_{1}, V_{2}, V_{3}$, with the property that any two are a basis and $V_{1}+V_{2}+V_{3}=0$. Call the basis reduced if not all three vectors are in a pair of (opposing) quadrants. This is the sign condition. Then under the neighbor process of the type $V_{1} \rightarrow V_{1}-V_{3}, V_{2} \rightarrow V_{2}+V_{3}, V_{3} \rightarrow V_{3}$, all such reduced bases are connected. This is proved by a modification of Smith's continued fraction algorithm. (Research sponsored by the U. S. Army O.O.R.) (Received November $13,1952$.

\section{P. F. Conrad: On ordered division rings.}

Some well known properties of ordered groups are used to prove theorems on ordered division rings. A division ring $D$ can be ordered if and only if there exists a valuation $f$ of $D$ for which the residue class ring $R$ can be ordered so that all elements of the form $P+a_{1}^{2} \cdots a_{n}^{2}$ are positive, where $P$ is the prime ideal of $f$ and $a_{1}^{2} \cdots a_{n}^{2}$ is a unit. $D$ can be ordered so that $f$ is the natural valuation (i.e., the valuation ring is the set of all elements that are comparable to 1 or infinitely smaller than 1 ) if and only if the ordering of $R$ is Archimedean. For each division ring with valuation there exists a maximal immediate extension. These results are generalizations of those obtained by W. Krull for ordered fields (J. für Math. vol. 167 (1932) pp. 160-196). For each ordered division ring there exists an $a$-closed $a$-extension (i.e., extension that preserves the value group). Every ordered field can be embedded in the field of formal power series over the value group with real coefficients. An $a$-closed ordered field is completely determined by its value group. (Received November 10,1952.)

\section{C. W. Curtis: Noncommutative extensions of Hilbert rings.}

Let $S$ be a ring containing a ring $R$ in its center, having a common identity with $R$, such that $S$ is a finite $R$-module, and $S$ satisfies the ascending chain condition for $R$-submodules. It is proved that each prime ideal in $S$ is an intersection of primitive ideals if and only if $R$ is a Hilbert ring in the sense of Goldman [Math. Zeit. vol. 54 (1951) pp. 136-140]. If $R$ contains a field $K$, and if each field homomorphic to $R$ has finite degree over the image of $K$, then each primitive algebra homomorphic to $S$ is finite-dimensional over $K$. The results are applied to prove, for example, that every irreducible representation of the universal associative of a Lie algebra over a field $K$ of prime characteristic is finite-dimensional over $K$. (Received November 10,1952.)

158t. C. W. Curtis: On commutative linear transformations in infinite-dimensional spaces.

Let $A$ be a linear transformation (1.t.) acting in a vector space $M$ of countable dimension over an algebraically closed field $K$. It is proved that if $A$ is (i) locally algebraic and if (ii) for each $\lambda \in K$, whenever $x$ is in the range of $(A-\lambda I)^{n}$ for every positive integer $n$, there exists a vector $y$, also in the range of $(A-\lambda I)^{n}$ for every $n$, such that $y(A-\lambda I)=x$, then each 1.t. commutative with every 1.t. commutative with $A$ is in the closure in the finite topology of the polynomial algebra $K[A]$. This result includes as a special case the theorem of Wedderburn which states that if $M$ is finitedimensional over $K$, then a 1.t. commutative with every 1.t. commutative with a given 1.t. $A$ is a polynomial in $A$. The proof is in part an application of Prüfer's well known 
decomposition theorem for countable primary abelian groups. Examples are given which show that the theorem is false if condition (i) is omitted, and that the method of proof fails if condition (ii) is not satisfied. (Received November 10, 1952.)

159. Gerard Debreu and I. N. Herstein: Nonnegative square matrices.

In a series of three papers in the Sitzungsberichte der Berliner Akademie, 1908, 1909 , and 1912, Frobenius obtained some very powerful theorems about the form of the largest (in absolute value) characteristic roots of matrices all of whose elements were nonnegative. These asserted, among other things, that for a certain fundamenta type of nonnegative matrix the largest characteristic root in absolute value was actually positive, simple, and had associated with it a positive eigenvector. In the present paper there is given a simple derivation of the key Frobenius' results. At the same time these are somewhat sharpened. Using these theorems the nonnegativity properties of $(s I-A)^{-1}$ are characterized for large $s$ where $A$ is a nonnegative matrix. A necessary and sufficient condition is given that the powers $A^{n}$ of the matrix $A$, having complex elements, converge as $n \rightarrow \infty$. Of late these types of results have found many applications in mathematical economics; the results of this paper seem to encompass most of the theorems that have been used in that discipline so far. General techniques are given to obtain further results along these lines. (Received November 14,1952.)

\section{D. O. Ellis: Remarks on Boolean functions.}

One considers functions $f(x)=(a \wedge x) \bigvee\left(b \wedge x^{\prime}\right)$ in a Boolean algebra $B$. The special case is that when $b \leqq a$. The principal remarks are: 1 . The set of Boolean functions on $B$ forms a ring of transformations on $B$ in which the group of multiplicative units is precisely the group of autometrized motions of $B$. The constant functions form an ideal in this ring and the resulting factor ring is isomorphic to the associated Boolean ring of $B$. 2. $f(x)$ maps $B$ onto $[a \wedge b, a \bigvee b]$.3. Let $f(x)$ be in the special case and let $B_{f}$ denote the space of the orbital topology induced by $f(x)$. Then the principal ideal of $a \oplus b$ is a group of homeomorphisms of $B_{f}$ onto itself and the join of orbital topologies for the special case is the strongest $T_{1}$ topology in $B$. One also considers the minimazation of the "triangle function" of $B$ and the fitting of graphs of Boolean functions to finite point sets in $B^{2}$. (Received November 7, 1952.)

\section{Melvin Henriksen: Rings of entire functions of finite order.}

Let $R_{\lambda}, R_{\infty}, R$ denote respectively the ring of entire functions of order $\leqq \lambda$, the ring of entire functions of finite order, and the ring of entire functions. In 1940, Helmer (Duke Math. J. vol. 6, pp. 345-356) showed that every finitely generated ideal of $R$ is principal, or equivalently, if $f, g$ in $R$ have no zeros in common, there exist $s, t$ in $R$ such that $s f+t g=1$. He asked if this held in $R_{\infty}$. A negative answer to this question existed already in 1936 (Proc. London Math. Soc. vol. 40, p. 256). In this paper, sufficient conditions that the residue class field of a maximal ideal of $R_{\lambda}$ or $R_{\infty}$ be isomorphic to the complex field are given and the existence of nonmaximal prime ideals is shown. The tools developed in a paper by the author (Pacific Journal of Mathematics vol. 2, pp. 179-184) on the ideal structure of $R$ are used. (Received November 12, 1952.)

162. S. T. Hu: Singular homology theory of associative algebras with unity elements. 
Let $W$ be any given associative algebra over the real field with a unity element $e$. By means of the algebras $A_{n}$ of continuous real functions defined on the unit $n$-simplex $\Delta_{n}$ in the $(n+1)$-dimensional euclidean space, $n=1,2, \cdots$, a semisimplicial complex $S(W)$ in the sense of Eilenberg and Zilber is defined in the present paper called the singular complex of the algebra $W$. The homology and cohomology groups of the complex $S(W)$ are defined to be the singular homology and cohomology groups of the algebra $W$. For each two-sided ideal $J$ of the algebra $W$, an associated subcomplex $\sigma(J)$ of $S(W)$ is defined. The relative homology and cohomology groups of $S(W)$ modulo $\sigma(J)$ are defined to be the relative singular homology and cohomology groups of the algebra $W$ modulo the ideal $J$. If $W=C(X)$ is the algebra of all continuous real functions defined on a compact Hausdorff space $X$, then the singular complex $S(X)$ of the space $X$ is simplicially isomorphic with the singular complex $S(W)$ of the algebra $W$, that is to say, there is a simplicial map $h: S(X) \rightarrow S(W)$ which maps $S(X)$ onto $S(W)$ in a one-to-one fashion. If $A$ is any closed subspace of $X$ and $J$ denotes the ideal of $W$ consisting of the elements $w$ with $w(A)=0$, then $h$ maps $S(A)$ onto $\sigma(J)$. This implies that the relative singular homology and cohomology groups of $X$ modulo $A$ are isomorphic with the corresponding groups of $W$ modulo $J$. (Received November 10, 1952.)

163. Arno Jaeger: On rings of differential operators over fields of prime number characteristic.

Let $D$ be an iterative differentiation in the sense of $\mathrm{F}$. K. Schmidt (cf. J. Reine Angew. Math. vol. 190 (1952)) of a field $F$ of characteristic $p \neq 0$ with the property $D x=1, D^{n} x=0\left(1<n<p^{i}\right)$ for a certain element $x \in F$. Let $F_{i}$ be the field of all constants of orders $\geqq i$ in $F$. Then, according to a basis theorem, the additive group $F^{+}$ of $F$ can be regarded as a vector space of dimension $p^{i}$ over $F_{i}$, the basis elements being the $x^{n}\left(0 \leqq n<p^{i}\right)$. Let $R_{i}$ be the ring of endomorphisms of $F^{+}$of the type $\alpha$ $=\sum_{n=0}^{p^{i-1}} a_{n} D^{n}\left(a_{n} \in F\right)$ where $\alpha y\left(\alpha \in R_{i}, y \in F\right)$ and $\alpha+\beta\left(\alpha, \beta \in R_{i}\right)$ are defined in the usual way and $\alpha \beta$ by $\alpha \beta=\sum_{n} a_{n} D^{n} \sum_{m} b_{m} D^{m}=\sum_{i} \sum_{n} \sum_{k \leqq n, j} C_{i, k} a_{n}\left(D^{n-k} b_{j-k}\right) D^{j}$. If an element of $R_{i}$ is an automorphism of $F^{+}$, then the inverse automorphism is an element of $R_{i}$, too. Every element of $R_{1}$ can be decomposed in a product of linear factors left multiplied by a unit. These and more complicated properties of $R_{i}$ (especially for $i>1$ ) are applied to solve linear differential equations in $F$ explicitly and to discuss the conditions for solvability in the nonhomogeneous case. (Received November 17, 1952.)

\section{4t. R. V. Kadison: Infinite general linear groups.}

The uniformly closed, normal subgroups of the general linear group (group of all invertible operators) of the various types of factors are studied. In the case of a factor of type $\mathrm{II}_{1}$, the determinant function comes into play, and it is shown that each closed, noncentral, normal subgroup of the general linear group of such a factor is the complete inverse image under the determinant map of some closed subgroup of the positive reals. The general linear group of a factor of type III contains no proper, closed, noncentral normal subgroups as is the case with the determinant 1 subgroup in a factor of type $\mathrm{II}_{1}$. The uniform closure in the general linear group of the set of invertible operators in a factor of type $\mathrm{I}_{\infty}$ or $\mathrm{II}_{\infty}$ which act as the identity on the orthogonal complement of some subspace of finite relative dimension is a proper, closed, noncentral, normal subgroup. In a factor of type $\mathrm{I}_{\infty}$ each closed, noncentral normal subgroup of the general linear group is the direct sum of the group just defined and the nonzero scalars. There are results which indicate that the same situation 
prevails in a factor of type $\mathrm{II}_{\infty}$, but the complete result is not obtained in this case. (Received November 13, 1952.)

\section{Irving Kaplansky: Dual modules over a valuation ring. II.}

This paper is chiefly devoted to locally linearly compact modules over a complete discrete valuation ring. After the requisite duality is set up, sundry structure theorems are proved; in particular, modules annihilated by $p^{2}$ admit complete analysis. Stated for a locally compact abelian group $G$ satisfying $p^{2} G=0$, the result asserts that $G$ is a direct sum of a discrete group, a compact group, and a third group which is characterized by a certain pair of dual vector spaces over the integers mod $p$. If $G$ satisfies the second axiom of countability, the vector spaces have countable dimension and the inner product between them is unique by a theorem of Mackey; this recaptures a theorem of Vilenkin. (Received November 13, 1952.)

\section{Erwin Kleinfeld: Simple alternative rings. 11.}

Let $R$ be a simple alternative ring, not a nil ring. Then using methods developed by R. H. Bruck and the author (see Proc. Amer. Math. Soc. vol. 2 (1951) pp. 878890 ) it is shown that the fourth power of every commutator of $R$ is in the nucleus of $R$. If $R$ is not associative then its nucleus and center coincide. A. A. Albert's result on simple alternative rings with nontrivial idempotents (see Canadian Journal of Mathematics vol. 4 (1952) pp. 129-135) reduces the situation to the case where the nilpotent elements can be shown to form an ideal. The final result is that $R$ is either associative or a Cayley-Dickson algebra of order eight over its center. (Received November 13, 1952.)

167. W. J. LeVeque: On the distribution modulo 1 of sequences involving periodic functions.

Suppose that $\phi \in C^{3}$ is periodic, of period $\omega$, and that both $\phi$ and its derivative $\phi^{\prime}$ are functions which have only finitely many zeros in $(0, \omega)$, and which have no zeros in common with their respective first derivatives. Then it is shown, using the method developed in earlier papers, that the sequence $\left\{u_{n} \phi\left(v_{n} x-c\right)\right\}$ is uniformly distributed (u.d.) modulo 1 for almost all $x \in(0, \omega)$ if either $(\mathrm{A})\left\{u_{n}\right\}$ is a positive lacunary sequence and $\left\{v_{n}\right\}$ is a positive nondecreasing sequence, or $(B)\left\{v_{n}\right\}$ is a positive lacunary sequence and $\left\{u_{n}\right\}$ is a positive nondecreasing sequence such that $n^{\epsilon}=O\left(u_{n}\right)$ for some $\epsilon>0$. Also, bounds are given for the associated exponential sum in the two cases. A corollary of case (A) is that the complex numbers $\xi, \xi^{2}, \cdots$, when reduced modulo 1 and modulo $i$, are uniformly distributed over the unit square $0 \leqq \operatorname{Re} z<1,0 \leqq \operatorname{Im} z<1$ for almost all complex $\xi$ with $|\xi|>1$. This extends a result due to Koksma. If neither $\left\{u_{n}\right\}$ nor $\left\{v_{n}\right\}$ is lacunary, the method fails for general periodic $\phi$, but it is shown that the following sequences are u.d. $(\bmod 1)$ for almost all $x \in(0,2 \pi):$ (C) $\left\{a n^{j} \cos \left(b n^{k} x-c\right)\right\}$, where $b$ and $k$ are positive integers with $j \geqq k$, and (D) $\left\{u_{n} \cos \left(u_{n} x-c\right)\right\}$, where $u_{n}$ is an increasing sequence of positive integers. (Received October 31, 1952.)

\section{8t. W. J. LeVeque: On uniform distribution modulo $\Delta$.}

Let $\Delta$ be a subdivision of the interval $(0, \infty): \Delta=\left(z_{0}, z_{1}, \cdots\right)$, where $0=z_{0}$ $<z_{1}<\cdots$ and $\lim _{n \rightarrow \infty} z_{n}=\infty$. For $z_{n-1} \leqq x<z_{n}$, put $[x]_{\Delta}=z_{n-1}, \delta(x)=z_{n}-z_{n-1}$, and $\langle x\rangle_{\Delta}=\left(x-[x]_{\Delta}\right) / \delta(x)$, so that $0 \leqq\langle x\rangle_{\Delta}<1$. Then the positive increasing sequence $\left\{x_{k}\right\}$ may be said to be uniformly distributed modulo $\Delta$ if the sequence $\left\{\langle x\rangle_{\Delta}\right\}$ is u.d. $(\bmod 1)$. Various criteria are given for uniform distribution $(\bmod \Delta)$, including ex- 
tensions of Fejer's theorem for sequences $\left\{x_{k}\right\}$ which increase slowly relative to $\left\{z_{n}\right\}$ (i.e., such that $\left[x_{k}\right]_{\Delta}$ is constant for long blocks of indices $k$ ), and the assertion that if $\delta(x)$ is a nonincreasing function such that $\delta(x)=O\left(x^{-1}\right)$, then $\{k \theta\}$ is u.d. $(\bmod \Delta)$ for almost all $\theta>0$. Finally, a transfer theorem is proved, making it possible to deduce uniform distribution $\left(\bmod \Delta_{2}\right)$ from uniform distribution $\left(\bmod \Delta_{1}\right)$ in certain cases. (Received October 31, 1952.)

169t. T. S. Motzkin and Olga Taussky: Pairs of matrices with property L. II.

A large class of pairs $A, B$ of $n \times n$ matrices with property $\mathrm{L}$ is characterized in a manner different from the one introduced originally (see T. S. Motzkin and O. Taussky, Bull. Amer. Math. Soc. vol. 58 (1952) p. 41 and Trans. Amer. Math. Soc. vol. 73 (1952) pp. 108-114). Consider the pencil $P$ of matrices $\lambda A+B$, and in particular the matrices with multiple characteristic roots in $P$. Assume that $P$ is not singular, i.e. not every matrix in $P$ has a multiple characteristic root. Then there are $n(n-1)$ values of $\lambda$ for which the corresponding matrix has a multiple characteristic root, namely the roots of the discriminant of $|\lambda A+B+x I|=0$. We say that the pair of matrices $A, B$ has property $\mathrm{D}$ if all these roots are double. It is proved that a pair of matrices $A, B$ with property $\mathrm{D}$ which generate a pencil, no matrix in which has a characteristic root of multiplicity $\geqq 3$ nor two different double characteristic roots, has property L. (Received November 13, 1952.)

170. Alex Rosenberg: The number of irreducible representations of simple rings with no minimal ideals.

An associative ring is said to be simple if it is semi-simple and has no proper twosided ideals. A simple ring is called a Zorn ring if every non-nil left ideal contains a nonzero idempotent. Let $A$ be a simple Zorn ring with no minimal ideals, unit element, and exactly $\aleph_{0}$ elements. Then it is shown that $A$ admits precisely $2 \aleph_{0}$ distinct (i.e. non-isomorphic) faithful irreducible modules. Further let $L$ be the ring of all linear transformations on a vector space of dimension $\mathbb{N}$ over a division ring of at most $\aleph$ elements, and let $F$ be the maximal two-sided ideal in $L$. Then it is shown that $L-F$ admits precisely $2 \mathbf{}$ distinct, irreducible modules. (Received November 12, 1952.)

\subsection{R. D. Schafer: A generalization of a theorem of Albert.}

A. A. Albert's recent theorem, that any finite power-associative division ring of characteristic $p>5$ is a field, is generalized as follows. Let $R$ be a finite power-associative ring without elements of additive order 2,3 , or 5 . If every element $a$ of $R$ satisfies an equation of the form $a^{n(a)}=a, n(a)$ an integer $>1$, then either (i) $R$ is a direct sum of finite fields, or (ii) the attached commutative ring $R^{+}$contains an ideal which is the unique 3-dimensional classical central simple Jordan algebra without nilpotent elements $\neq 0$ over some finite field; in this latter case, $R^{+}$is a direct sum of fields and such 3-dimensional algebras (over possibly different finite fields). (Received November 3,1952 .)

172. L. M. Weiner: A factorization theorem concerning sums of homogeneous functions.

In this paper is begun the study of the ring $R$ of functions which are representable as sums of homogeneous functions. Any element of $R$ is $F=F_{n}+F_{n-1}+\cdots+F_{0}$, where $F_{i}$ is a real-valued, continuous, homogeneous function of degree $i$ of $m$ real 
variables. This ring $R$ is not an integral domain but contains the polynomial integral domain as a proper subset. Associated with each point $p$ of the region of definition of the elements of $R$ and each $F$ of $R$ is the polynomial $P_{F}(p)=F_{n}(p) x^{n}+F_{n-1}(p) x^{n-1}$ $+\cdots+F_{0}(p)$. In general, an element $F$ of $R$ cannot be factored uniquely into irreducible factors. The following theorem is established. If $F$ is an element of $R$ defined in a region $R$ such that the discriminant of $P_{F}(p)$ is distinct from zero and $F_{n}(p)$ and $F_{n-1}(p)$ are not both zero for any point $p$ of $R$, then $F$ is factored uniquely into irreducible factors in $R$ aside from the order of the factors and the units. (Received November 12, 1952.)

\section{George Whaples: Additive polynomials. II.}

Let $k$ be a field with characteristic $p$, no inseparable extensions, and infinitely many elements. All decompositions (1) $u(x)=v(f(x))+g(x)$ and (2) $u\left(x^{p^{m}}\right)=h(w(x))$, where $u, v, w$ denote arbitrary polynomials over $k$ with constant term zero and $f, g, h$ additive polynomials $(=a . p .=p$-polynomials as defined by $O$. Ore, Trans. Amer. Math. Soc. vol. 35 (1933) pp. 559-584), are consequences of the following maximal ones. In (1), $f(x)$ may be chosen as the least degree a.p. whose zeros are the set of all $\Gamma$ in the algebraic closure of $k$ with $u(x+\Gamma)=u(x)+u(\Gamma)$. In $(2), h(x)$ may be chosen as the least degree a.p. for which an identity (3) $h\left(x^{p^{n}}\right)=\sum_{\nu} u\left(l_{\nu}(x)\right)$ holds. If furthermore $k$ has one extension of degree $p$ and for each $n$ at most one extension of degree $n$, then (3) may be replaced by the condition: $h(x)=$ the least degree a.p. with $h\left(K^{+}\right)$ =additive group generated by $u\left(K^{+}\right)$for all finite extensions $K$ of $k$. (Received November 24,1952 .)

\section{ANALysis}

\section{W. G. Bade: Unbounded spectral operators. Preliminary report.}

N. Dunford has shown (see Bull. Amer. Math. Soc. Abstract 59-2-185) that if $S=\int \lambda E(d \lambda)$, where $E(\beta)$ is a compact projection valued measure on the Borel subsets $\beta$ of the complex plane, then a bounded operator $T$ commuting with all the $E(\beta)$ is a spectral operator (i.e. satisfies $\sigma(T, E(\beta) X) \subset \bar{\beta}$ for each $\beta$ ) if and only if $T=S+N$ where $N$ is a bounded quasinilpotent commuting with $S$. The corresponding problem for unbounded operators is investigated where $E(\beta)$ is a measure on the complex sphere $K$ and $S x=\lim \int_{c_{n}} \lambda E(d \lambda) x, C_{n}=\{\lambda ;|\lambda| \leqq n\}$ where this limit exists. A closed unbounded operator $T$ is a spectral operator (i.e. $\sigma(T, E(\beta) X \subset \bar{\beta}$, the closure of $\beta$ in $K$ ) in case $N$, defined by $N x=T x-S x$, is bounded on the dense set $\{x ; x=E(\beta) y$, $y \in X, \beta$ bounded $\}$. However, examples are constructed to show $T$ may be an unbounded spectral operator with (a) $N$ unbounded with no resolvent set, (b) $N$ bounded but not quasinilpotent in $X$. An operational calculus for $T$ and $S$ is investigated. (Received December 15, 1952.)

175. R. G. Bartle: Solutions of equations in Banach spaces. Preliminary report.

A study is made of the equation $\left(^{*}\right) v=H(u)$, where $H$ maps a Banach space $\mathfrak{U}$ into $\mathfrak{B}$, and has on $\mathfrak{U}$ a Fréchet differential mapping onto $\mathfrak{B}$. The following results are cited. Given $v_{0}$ such that $\left(^{*}\right)$ has $k$ distinct solutions, conditions are given to assure that (1) points in a neighborhood of $v_{0}$ have (1a) at least $k$ distinct solutions, or (1b) exactly $k$ solutions, and that (2) every point in $H(\mathfrak{U})$ has exactly $k$ distinct solutions. If the inverse image under $H$ of a line segment in $\mathfrak{B}$ is compact, then $H$ maps onto $\mathfrak{B}$; if, in addition, $H$ is locally $1-1$, then $H$ is a homeomorphism of $\mathfrak{U}$ onto $\mathfrak{B}$. If $\mathfrak{I}$ is a compact metric space and $\Gamma_{0}=\left\{x_{0}(t) ; t \in \mathfrak{I}\right\}$ is a curve in $\mathfrak{U}$ which $H$ sends into $v_{0}$, then for $v_{1}$ sufficiently near $v_{0}$ there will be a curve $\Gamma_{1}=\left\{x_{1}(t) ; t \in \mathfrak{T}\right\}$ 
mapping into $v_{1}$ such that $x_{1}(t)$ is uniformly close to $x_{0}(t)$, for $t \in \mathfrak{T}$. (Received November 13, 1952.)

176t. S. D. Bernardi: On an inequality of Prawitz. Preliminary report.

Recent results of the author (see Two theorems on schlicht functions, Duke Math. J. vol. 19 (1952); A survey of the development of the theory of schlicht functions, Duke Math. J. vol. 19 (1952)) are improved through the application of the inequality $\sum_{n=1}^{\infty}(2 n-\alpha)\left|b_{n}\right|^{2} / \alpha \leqq 1$, where $f(z)=z+\sum_{n=2}^{\infty} a_{n} z^{n}$ is regular and schlicht for $|z|<1$ and $(f(z) / z)^{-\alpha / 2}=1+\sum_{n=1}^{\infty} b_{n} z^{n}, \alpha$ real. The inequality is applied to obtain information in the form of inequality relations concerning functions $f(z)$ with coefficients $a_{n}$ belonging to special algebraic fields. The methods are similar to those used in the two aforementioned papers. (Received November 4, 1952.)

\section{7t. H. D. Block: Construction of solutions and propagation of} errors in nonlinear problems.

It is intended that the results of this paper provide usable theoretical tools for numerical computation of the solutions of a class of nonlinear problems. If $X$ is an additive group with a metric which is invariant under translation, then we call $X$ a $b$-space. Let $X$ and $Y$ be $b$-spaces and let $F$ map $X$ into $Y$. With certain assumptions concerning $F$, the equation $F x=y$ has, for each $y$ in $Y$, a unique solution $x$, the iterative construction of which is presented. Bounds on the error due to using only a finite number of iterations are presented, as well as some other useful estimates. Bounds on the errors involved due to using the perturbed equation: $(F+f)(x+\xi)$ $=(y+\eta)$ are also given. If $T$ is an arbitrary set and $G$ maps $T \times X$ into $Y$ then, under suitable assumptions on $G$, the equation $G(t, x)=0$ defines an implicit function $x=x(t)$. The iterative determination of $x(t)$ is also treated. Finally an iterative method for the eigenvalue problem $F x=\lambda x$, where $X$ and $Y$ are now the same normed vector space, is also presented. (Received November 13, 1952.)

178t. F. F. Bonsall and Morris Marden: The zeros of rational functions with self-inversive polynomial factors.

This paper generalizes the authors' previously announced results [Bull. Amer. Math. Soc. Abstract 58-2-157]. Let $M[\Phi, T]$ denote the total multiplicity of the zeros of a function $\Phi$ in the region $T$ and $N[\Phi, T]$ the number of distinct zeros of $\Phi$ in $T$. The principal result of this paper may be stated as follows: Let $\phi=(f g) /(F G)$, where $f$ and $F$ are self-inversive polynomials (i.e., symmetric in the unit circle $C$ ), where $g$ is a polynomial with all its zeros inside $C$ and $G$ one with all its zeros outside $C$; and where degree $(f g)>$ degree $(F G)$; then, for the derivative $\phi^{\prime}$ of $\phi, M\left[\phi^{\prime},|z|>1\right]$ $=M[\phi,|z|>1]+N[1 / \phi,|z| \geqq 1]$. Thus, if $h(z)$ is any polynomial having all its zeros in $C$ and if $\psi(z)=h(z) \phi(z)$, then $M\left[\psi^{\prime},|z|>1\right]=M\left[\phi^{\prime},|z|>1\right]$. These results are also extended to certain cases where degree $(f g)=\operatorname{degree}(F G)$. (Received November 26, 1952.)

179. H. J. Bremermann: The kernel function and invariant metric in domains of regularity.

A domain of existence of an analytic function of complex variables is denoted as a "domain of regularity." Any domain $G$ possesses an "envelope of regularity" $R(G)$. The Bergman kernel function $K_{G}(z, \xi)\left(z=\left(z_{1}, z_{2}\right), \zeta=\left(\zeta_{1}, \zeta_{2}\right)\right)$ for any domain $G$ is 
analytic in $R_{z}(G) \times R_{\bar{\gamma}}(G)$. If $K_{G}(z, \bar{z})$ becomes infinite at all boundary points of $G$, then $G$ is a domain of regularity. (Corollary: The domains $K_{G}(z, \tilde{z})<M$ are domains of regularity.) Examples show that for an arbitrary domain of regularity $R$ the domain $R_{z} \times R_{\bar{\zeta}}$ is not necessarily the domain of existence of the kernel function $K_{R}(z, \bar{\zeta})$. But if $R$ can be approximated by a sequence of domains of regularity containing $\bar{R}$, then $R_{z} \times R_{\bar{\zeta}}$ is the domain of existence of $K_{R}(z, \bar{\zeta})$. If in addition the boundary of $R$ is "smooth" or if $R$ is an analytic polyeder, then $K_{R}(z, \bar{z})$ becomes infinite everywhere at the boundary of $R$. In this case all boundary points of $R$ are infinite points with respect to the Bergman metric. On the other hand, if one can introduce in a domain $G$ a Kähler metric with analytic coefficients in such a way that all boundary points are "infinite," then $G$ is a domain of regularity. (Received November 17, 1952.)

180. H. D. Brunk: On functions having isolated singularities on the positive real axis. Preliminary report.

In a strip region, $\Delta$, let $f(z)$ be holomorphic except for isolated singularities at points $z=\lambda_{n}$ (real), $n=1,2, \cdots, 0<\lambda_{n} \uparrow \infty$. If $f(z)$ is suitably restricted on the boundary of $\Delta$, an elementary observation involving the residue theorem yields a function $F(s)$ represented by the partial sums $\sum_{k-n}^{n} a_{k} \exp \left(-\lambda_{k} s\right)$ asymptotically with respect to a sequence of positive constants related to the growth of $f(z)$ in $\Delta$; here $a_{k}$ is the residue of $f(z)$ at $z=\lambda_{k}$. Theorems on asymptotic Dirichlet series ((1) Mandelbrojt, Ann. École Norm. (3) vol. 43 (1946) pp. 351-378; (2) Mandelbrojt and Brunk, Duke Math. J. vol. 18 (1951) pp. 297-306; (3) Brunk, Bull. Amer. Math. Soc. vol. 55 (1949) pp. 204-212) then give theorems relating the magnitudes of the residues of $f(z)$ to its growth in $\Delta$. For example, one can say, roughly, that if $f(z)$ fails to grow sufficiently rapidly in $\Delta$, the fundamental inequality of (1) shows that $\lim \sup _{n \rightarrow \infty}\left(\log \left|a_{n}\right|\right) / \lambda_{n}$ $<\infty$ (if $\lim \inf _{n \rightarrow \infty} \lambda_{n+1}-\lambda_{n}>0$ ). Similarly, the theorem of (2) has application to a situation in which at each point $z=\lambda_{k}$ one or the other of two functions holomorphic in a certain right half-plane except for isolated singularities at points $z=\lambda_{k}$ has residue 0 . (Received November 12, 1952.)

\section{1t. P. L. Butzer: On the extensions of Bernstein polynomials to the} infinite interval.

Let the function $f(x)$ be defined over the infinite interval $0 \leqq x<\infty$. Szász [Jour. Research Nat. Bur. Stand. vol. 45 (1950) pp. 239-245] introduced the transform $P^{f}(u ; x)=e^{-u x} \sum_{v=0}^{\infty}\left[(u x)^{v} / v !\right] f(v / u)$, for which $\lim _{u \rightarrow \infty} P^{f}(u ; x)=f(x)$ uniformly in $(0, \infty)$ if $f(x)$ is continuous in $(0, \infty) . P^{f}(u ; x)$ is the analogue for the interval $[0,1]$ of the familiar Bernstein polynomials. The author considers a generalization of $P^{f}(u ; x)$, namely the transform $W^{f}(u ; x)=u e^{-u x} \sum_{v=0}^{\infty}\left[(u x)^{v} / v !\right] \int_{v / u}^{(v+1) / u} f(s) d s$ where $f(x)$ is Lebesgue integrable over the interval $0 \leqq x<\infty$, i.e. $f(x) \in L(0, \infty)$. Let $M^{f}(x)=\sup _{0<s<\infty}(1 /(s-x)) \int_{x}^{s}|f(y)| d y$. If $f(x)$ and $|f(x)|^{p}, p>1$, both belong to $L(0, \infty)$, then it is shown that (i) $\lim _{u \rightarrow \infty} W^{f}(u ; x)=f(x)$ almost everywhere in $(0, \infty)$, (ii) there exists a function $M^{f}(x)$ in the space $L^{p}$ such that $\sup _{u>0}\left|W^{f}(u ; x)\right| \leqq 3 M^{f}(x)$ almost everywhere in $(0, \infty)$. As a corollary $\int_{0}^{\infty}\left|W^{\prime}(u ; x)-f(x)\right| p d x \rightarrow 0$ as $u \rightarrow \infty$. (Received November 13, 1952.)

182. Lamberto Cesari: On the analytical representation problem of Frechet surfaces.

After a series of research (C. B. Morrey, J. W. T. Youngs, E. J. Mickle) L. Cesari proved the following theorem: Given any continuous mapping of finite Lebesgue area 
$(T, A): p=T(w), w=(u, v) \in A$, from a closed 2-cell $A$ of the $w$-plane into the $p$-space $E_{3}, p=(x, y, z)$, there is Frechet equivalent mapping $\left(T^{\prime}, B\right): p=T^{\prime}(w), w \in B$, from the unit circle $B$ into $E_{3}, T^{\prime}(w)=[x(u, v), y(u, v), z(u, v)]$, where the functions $x, y, z$ have a.e. in $B^{0}$ ordinary first partial derivatives $L^{2}$-integrable in $B^{0}$; the Lebesgue area is given by the classical integral, and $E=G, F=0$ a.e. in $B^{0}$. The author has given a new proof of the theorem above, under more general conditions, where no use is made of the Dirichlet integral and of the topological structure properties of continuous mappings. Consistent use is made of tangential properties of mappings of finite Lebesgue area (L. Cesari, Comment. Math. Helv. vol. 22 (1949) pp. 1-17) and of the existence and properties of an everywhere dense system of rectifiable contours (L. Cesari, Bull. Amer. Math. Soc. vol. 57 (1951) p. 168; R. E. Fullerton, Ibid. vol. 58 (1952) p. 57, and p. 180). The set $A$ is supposed to be any of the following sets: any open set $A^{\prime}$; any finite sum $A^{\prime \prime}$ of disjoint finitely connected Jordan regions; any subset $A^{\prime \prime \prime}$ of a set $A^{\prime \prime}$ open in $A^{\prime \prime}$. The set $B$ is supposed to be any set topologically equivalent to $A$. (Received November 12,1952.)

183. J. M. Danskin: An extension of the Brown-Robinson iterative process for finding the value of a game.

Let $M(x, y)$ be a continuous function defined on the unit square. We define sequences $\left\langle x_{n}\right\rangle$ and $\left\langle y_{n}\right\rangle$ as follows: $\left\langle x_{n}\right\rangle: x_{0}$ is arbitrary; $x_{n}$ maximizes the sum $\sum_{k=0}^{n-1} M\left(x, y_{k}\right)(n \geqq 1) ;\left\langle y_{n}\right\rangle: y_{0}$ is arbitrary; $y_{n}$ minimizes the sum $\sum_{k=0}^{n-1} M\left(x_{k}, y\right)$ $(n \geqq 1)$. It is proved that $\lim _{n \rightarrow \infty}(1 / n) \sum_{k=0}^{n-1} M\left(x_{n}, y_{k}\right)=\lim _{n \rightarrow \infty}(1 / n) \sum_{k=0}^{n-1} M\left(x_{k}, y_{n}\right)=v$, where $v$ is the "value of the game" in the sense of the theory of games. This generalizes a result of Julia Robinson, proved for matrices (Ann. of Math. vol. 54 (1951) 296-301). The result remains valid when the unit square is replaced by the direct product of two compact spaces. (Received November 13, 1952.)

184. Jim Douglas, Jr.: On the expansion of measures in series.

Let $(X, S)$ be a measurable space, and let $\left\{z_{\alpha}\right\}$ be the class of all measures defined on $(X, S)$ each of which assumes the values zero and one only. Let measures $m_{1}$ and $m_{2}$ be absolutely continuous with respect to $n$ with density functions $f_{1}$ and $f_{2}$. Then the Kakutani quasi-metric is $\rho\left(m_{1}, m_{2}\right)=\int\left(f_{1} f_{2}\right)^{1 / 2} d n$. For each totally finite signed measure $m$, define $m_{*}^{*}=\sum \rho^{2}\left(m, z_{\alpha}\right) z_{\alpha}$. Various necessary and sufficient conditions for $m=m^{*}$ are given. As for ordinary Fourier series, there exist spaces $(X, S)$ and measures $m$ for which $m^{*} \neq m$. Conditions on $S$ are given to insure $m=m^{*}$ for all $m$ defined on $S$. (Received November 10, 1952.)

\section{Nelson Dunford: Spectral operators.}

An operator $T$ in a complex Banach space $X$ is called a spectral operator in case it has a resolution of the identity $E$ defined on the Borel sets in the plane. For such operators the vector valued function $(\xi I-T)^{-1} x$ has a maximal single-valued analytic extension $x(\xi)$ defined on an open set $\rho(x)$. The spectrum $\sigma(x)$ of $x$ is defined to be the complement of $\rho(x)$. The resolution of the identity is unique and satisfies the relation $E(\sigma) X=[x \mid \sigma(x) \subset \sigma]$, providing $\sigma$ is closed. A corollary is that $E(e)$ commutes with every bounded linear operator which commutes with $T$. The operator $S$ is said to be of scalar type in case it is spectral and satisfies $S=\int \lambda E(d \lambda)$. An operator $T$ is spectral if and only if it is the sum $T=S+N$ of a scalar type operator $S$ and a generalized nilpotent $N$ commuting with $S$. This canonical decomposition is unique and for functions $f$ analytic on the spectrum of $T, f(T)=\sum\left(N^{n} / n !\right) f f^{(n)}(\lambda) E(d \lambda)$. Commutative algebras of spectral operators are of the form $A=C(M) \oplus R$ where $M$ is the space of maximal ideals in $A$ and $R$ is the radical in $A$. (Received November 13,1952.) 
186. P. R. Garabedian and M. M. Schiffer: Convexity of domain functionals.

The second variations of the capacity $\gamma$, virtual mass $\alpha$, and principal frequency $\lambda$ of a region $D$ are derived rigorously for Hadamard shifts of the smooth surface $S$ of $D$ and for interior variations of $D$ in space of two and three dimensions. When $S$ is shifted along level surfaces of a harmonic function $u$, it is shown that the capacity $\gamma$ of $S$ is a convex function of $u$. The inverse square $1 / \lambda^{2}$ of the principal frequency of a domain $D$ obtained as the image of a fixed plane region by a conformal mapping $z+\epsilon f(z)$ is shown to be a convex function of $\epsilon$. Convexity theorems such as these are used to prove that domains for which certain combinations $q$ of capacity, virtual mass, and principal frequency are stationary actually minimize $q$ and are unique. For example, the result of Pólya and Szegö that $\lambda e^{\gamma}$ is a maximum for a circular disc is rederived, and it is shown that the virtual mass of an axially symmetric parallel flow past a surface $S$ enclosing two circular rings is a minimum when $S$ reduces to a vortex sheet spanning the two rings. (Received December 5, 1952.)

\section{Felix Haas: A theorem about differential equations on two- dimensional manifolds.}

Let $M$ be a closed, orientable, two-dimensional manifold. $V$, a vector field on $M$, defines a differential equation in a way which insures uniqueness. Let the singular points of $V$ form a compact set. Finally, let $C^{+}$be a semi-characteristic of the differential equation defined by $V$, and let $C$ be the set of $\omega$-limit points of $C^{+}$which we assume free of singular points. Then the following theorem holds: If $\bar{C}$ is dense anywhere on $M, M$ is a torus and $V$ is free of singular points. This is the best theorem possible in this direction since the work of Denjoy shows that if $M$ is a torus and $V$ is free of singular points, the set $\bar{C}$ may certainly be dense in some set $G$ of $M$. The proof proceeds along the following lines: If $\bar{C}$ does contain a closed curve or a curve of infinite arc length which is a characteristic of the orthogonal vector field, it is shown that $M$ is a torus and $V$ is free of singular points. If $\bar{C}$ does not contain such a curve, it is shown that $\bar{C}$ cannot be dense anywhere on $M$. (Received October 28, 1952.)

\section{Henry Helson: Lacunary sets of integers.}

Classical theorems of Sidon and of Zygmund on lacunary trigonometric series were proved under the hypothesis that the frequencies $n_{1}, n_{2}, \cdots$ occurring in the series satisfy a relation $n_{\nu+1} / n_{\nu} \geqq k>1$. Two conditions weaker than this one are given, one necessary for the truth of Sidon's theorem, the other sufficient for Zygmund's theorem. The proofs depend on theorems of Hardy and Littlewood. (Received November $10,1952$.

\section{9t. Alfred Huber: On the uniqueness of generalized axially sym-} metric potentials.

Let $G$ be a region lying in the half-plane $y>0$, the boundary of which contains a segment $S$ of $y=0$. Let $u(x, y)$ be a solution of $y \Delta u+k u_{y}=0$ ( $k$ an arbitrary real constant), defined in $G$, assuming the boundary value 0 on $S$. The results are: (1) For $k \geqq 1: u \equiv 0$ throughout $G$. (2) For $k<1: u=y^{1-k} v(x, y)$, where $v$ is analytic in $G+S$ and satisfies $y \Delta v+(2-k) v_{y}=0$. Inversely each function $u$ of this form satisfies the above conditions. The proof involves the use of the identification principle for solutions even in $y$ and analytic on $S$ (A. Weinstein, Discontinuous integrals and generalized 
potential theory, Trans. Amer. Math. Soc. vol. 63 (1948) p. 344) and a theorem on removable singularities on $y=0$. This work was done under contract N7onr 397(05). (Received November 21, 1952.)

\section{J. L. Kelley: Extension theorem for finitely additive measures.}

Let $p$ be a non-negative, monotonic function defined on a boolean algebra $\mathcal{A}$ and satisfying: $p(0)=0$ and (1) $p(x)+p(y) \geqq p(x \vee y)+p(x \wedge y)$ (for example, Lebesgue outer measure on the algebra of all subsets of the real numbers). Let $m$ be a nonnegative, additive function, defined on a subalgebra of $\mathcal{A}$, such that $m(x) \leqq p(x)$ for each $x$. Then there is a non-negative additive extension of $m$ to all of $\mathcal{A}$ which is also dominated by $p$. If condition (1) is replaced by (2) $p(x)+p(y) \geqq p(x \vee y)$, the corresponding theorem is false. (Received November 17, 1952.)

191t. M. S. Klamkin: Sums and asymptotic sums of series related to the harmonic series. II.

$\sum_{p=1}^{p}\left[S_{m, p} / p^{n}+S_{n, p} / p^{m}\right]=S_{m, p} S_{n, p}+S_{m+n, p}+\sum_{p_{m=1}}^{p}\left[\sum_{m, n, r \text { cyclic }} S_{m, p} S_{n, p} / p^{r}-S_{m, p} / p^{n+r}\right]$ $=S_{m, p} S_{n, p} S_{r, p}-S_{m+n+r, p}$, two identities of 2 nd and 3rd order respectively, are extended to the $N$ th order where $S_{m, p}=\sum_{1}^{p} 1 / p^{m}$. These identities are also valid if $S_{m, p}=\sum_{1}^{p} 1 / a_{p}^{m}$. If $T_{r, p}=\sum_{1}^{p} 1 / b_{p}^{r}$ we also have $\sum_{1}^{p}\left[S_{n, p} / b_{p}^{m}+T_{m, p} / a_{p}^{n}\right]=S_{n, p} T_{m, p}$ $+\sum_{1}^{p} 1 / a_{p}^{n} b_{p}^{m}$. This is also extended to higher order. The above identities are then specialized to obtain sums and asymptotic sums of series whose $p$ th terms are given by $(-1)^{p+1} S_{1, p} / p ;(-1)^{p+1} R_{1, p} / p ; p^{n} S_{1, p} ;$ and $S_{r, p}^{m} R_{s, p}^{n} / p$ where $R_{s, p}=\sum_{1}^{p}(-1)^{p+1} / p^{s}$. (Received November 13, 1952.)

\section{V. L. Klee, Jr.: Invariant extension of linear functionals.}

There is established a theorem on extension of linear functionals, subject to being majorized by a given positively homogeneous subadditive functional and to being invariant under a given set of linear transformations. As corollaries are obtained an improvement of the results of Agnew and Morse (Ann. of Math. vol. 39 (1938) pp. 20 30) and Woodbury (Bull. Amer. Math. Soc. Abstract 56-2-168) on invariant extension of functionals, and also the principal results of Dunford and Yood (Proc. Amer. Math. Soc. vol. 2 (1951) pp. 225-233) on existence of a common nonzero fixed point for the adjoints of a semi-group of bounded linear operators. Besides the previously known cases of commutative semi-groups and solvable or finite groups, the desired extension and existence theorems are established for compact groups of bounded linear operators. (Received November 14, 1952.)

193. G. G. Lorentz and D. G. Wertheim: Linear functionals on Köthe spaces.

Let $X_{C}=X$ be the ("Köthe") space of all real functions $f(t)$ such that $\|f\|$ $=\sup \int_{0}^{1}|f| c d t<+\infty$, where the supremum is taken for all $c(t) \in C$. Properties of $C$ are discussed which ensure that $X_{C}$ is a Banach lattice with some simple abstract properties. Let $X(B)$ be the space of all weakly measurable functions $\bar{f}(t)=\bar{f}_{t}$ from $(0,1)$ to a Banach space $B$ such that $\|\bar{f}\|=\|\| \bar{f}(t)\left\|_{B}\right\|_{x}<+\infty$. Under simple assumptions on $X$ and for separable $B$ the general form of a continuous linear functional $L(f)$ on $X(B)$ is given by $L(f)=\int_{0}^{1} \bar{F}_{t}\left(\bar{f}_{t}\right) d t$ where $\bar{F}_{t}$ is an element of the space $X^{*}\left(B^{*}\right)$. The proof uses operators of class $(b, 0)$ of Kantorovitch and is different from that given by Dieudonné [Canadian Journal of Mathematics vol. 3 (1951) pp. 129-139] for the special case $X=L^{p}$. (Received November 14,1952.) 
194. G. R. MacLane: Distortions of the Riemann surface of the inverse sine.

The Riemann surface of $z=\arcsin w$ may be split into "sheets" $S_{n}$, where each $S_{n}$ is a $w$-plane cut along all the real axis except for the interval $(-1,1)$. Let $S_{n}$ be distorted as follows: the two cuts, $(1,+\infty)$ and $(-\infty,-1)$, are pushed, remaining parallel to the real axis, into any two cuts parallel to the real axis. This distortion is limited by the following requirement: let $d$ be a constant, $0<d<\pi / 2$, and let there exist points $w_{n}$ such that in each distorted $S_{n}$ one cut lies in $\left|\arg \left(w-w_{n}\right)\right|<\pi / 2-d$ and the other cut lies in $\left|\arg \left(w_{n}-w\right)\right|<\pi / 2-d$. Then the distorted surface is parabolic and is the image of $|z|<\infty$ by an entire function $w=f(z)$. Assuming that $0 \in S_{0}$ is not a branch point of the surface, there exists a particular normalization of this map, $f(0)=0 \in S_{0}, f^{\prime}(0)=p_{0}+q_{0} i, p_{0}^{2}+q_{0}^{2}=1$, such that $f^{\prime}(z)$ has infinitely many distinct representations of the form $f^{\prime}(z)=\left(\exp \left(-a z^{2}\right)\right)\left\{c_{1} e^{r z} \pi_{1}(z)+c_{2} e^{s z} \pi_{2}(z)\right\}$, where $a, r$, and $s$ are real constants, $a \geqq 0$, and $\pi_{i}(z)=\Pi^{\prime}\left\{\left(1-z / b_{i, k}\right) \exp \left(z / b_{i, k}\right)\right\}$, with $\cdots b_{i,-k}$ $<b_{i,-k+1}<\cdots<b_{i,-1}<0<b_{i, 1}<\cdots<b_{i, k}<\cdots$ and the zeros of $\pi_{1}$ and $\pi_{2}$ are approximately interlaced: $b_{2, k-1}<b_{1, k}<b_{2, k+1}<b_{1, k+2}$. (Received November 12, 1952.)

195t. Hazleton Mirkil: Translation-invariant function algebras over compact groups. II.

Let $A$ be a function algebra (pointwise multiplication) which is "left-homogeneous" over a compact group $G$ in the sense of our previous abstract (Bull. Amer. Math. Soc. Abstract 58-6-642). Theorem: The following three lattices are isomorphic: (1) the left-quotients of $G$ by closed subgroups, (2) the left-invariant self-adjoint subalgebras of $Q(G)$, (3) the left-invariant self-adjoint closed subalgebras of $A$. These isomorphisms are the natural ones, e.g. the subalgebra corresponding to a given leftquotient $G / G_{0}$ consists of all functions which are constant on each left coset, or, equivalently, which admit the elements of $G_{0}$ as right periods. In particular, the theorem states that left-invariant self-adjoint subalgebras of $Q$ are automatically closed in $Q$ for all reasonable norms, and that left-invariant self-adjoint closed subalgebras $A_{0}$ of $A$ are generated by $A_{0} \cap Q$. (Received October 16, 1952.)

196t. Hazleton Mirkil: Translation-invariant function algebras over compact groups. III.

Let $A$ be left-homogeneous over compact $G$, and let $J$ be the ideal consisting of elements which vanish on neighborhoods of $e \in G$. $A$ is "of type C" if (1) for all $s \neq e$ there exists $x \in J$ with $x(s) \neq 0$, and (2) there exists $k>0$ with $\|x+y\|>k\|x\|$ for all $y \in J$ and all $x . A / \bar{J}$ is then a "local" algebra, i.e., it has a unique maximal ideal, and $A$ is completely determined by the restriction to $Q(G)$ of the mapping $A \rightarrow A / \bar{J}$. Conversely, given an abstract local banach algebra $P$ with maximal ideal $M$ and a homomorphism $h: Q \rightarrow P$ satisfying $h\left(u_{i j}\right) \equiv \delta_{i j}(\bmod M)$ for every irreducible matrix element $u_{i j}$, a condition which is clearly necessary, one can construct in a natural way a left-homogeneous algebra of type C. (Received October 16, 1952.)

197t. Hazleton Mirkil: Translation-invariant function algebras over compact groups. IV.

Let $G$ be a compact group and let $A$ be the finite linear combinations of continuous positive-definite functions. It is known that the pointwise product of two elements of $A$ belongs to $A$, and that $A$ has a norm (topologically unique) which makes it a complete normed algebra, in fact a "left-homogeneous" algebra over $G$. Theorem: The 
closure of the ideal $J$ in $A$ (see the preceding abstract) contains all elements of $A$ which vanish at $e$. Equivalently, every closed ideal lies in more than one maximal ideal unless it is itself maximal. When $G$ is the circle, $A$ is the absolutely convergent fourier series with $\left\|\sum c_{n} e^{i n t}\right\|=\sum\left|c_{n}\right|$; and for this case the theorem was first proved by Wiener. Kaplansky generalized Wiener's result to arbitrary commutative (even noncompact) groups $G$. (Received October 16, 1952.)

198t. Hazleton Mirkil: Translation-invariant function algebras over compact groups. V.

Let $G$ be the multiplicative group of complex numbers of absolute value one, and let $A$ be a homogeneous algebra over $G$. Then $A$ must have one of two mutually exclusive separation properties: it must be either "interval-quasi-analytic" in the sense of Mandelbrojt (definition: every $x \in A$ constant on an open subset of $G$ is constant on $G$ ), or else "regular" in the sense of Silov (definition: every two disjoint closed subsets $S_{0}$ and $S_{1}$ of $G$ can be separated by some $x \in A, x \equiv 0$ on $S_{0}, x \equiv 1$ on $S_{1}$ ). This dichotomy does not hold for homogeneous algebras $A$ over an arbitrary compact group $G$. (Received October 16, 1952.)

199t. T. S. Motzkin and J. L. Walsh: Least pth power polynomials on a real finite point set.

Let $E$ be the set of $m$ real distinct points $\left\{x_{1}, x_{2}, \cdots, x_{m}\right\}$, and for variable $\xi_{1} \leqq \xi_{2} \leqq \cdots \leqq \xi_{n}$ let the polynomial $T_{n}(x) \equiv \prod_{1}^{n}\left(x-\xi_{i}\right)$ minimize $\sum_{1}^{m} \mu_{i}\left|T_{n}\left(x_{i}\right)\right|^{p}$, $\mu_{i}>0,0<n<m$. For $p>1, T_{n}(x)$ is unique and (1) $x_{0}^{\prime}<\xi_{1}<x_{1}^{\prime}<\cdots<\xi_{n}<x_{n}^{\prime}$ where the $x_{i}^{\prime}$ belong to $E$. (2) For $0<p<1$ every $T_{n}(x)$ has distinct roots $\xi_{i}$ in $E$. For $p=1$ there exist fundamental polynomials $T_{n}^{(k)}(x)$ with distinct roots in $E$ such that the set $T_{n}(x)$ is precisely the set $\left\{\sum_{k} \lambda_{k} T_{n}^{(k)}(x), \sum \lambda_{k}=1, \lambda_{k} \geqq 0\right\}$; every $T_{n}(x)$ fulfills (3), namely (1) with the signs $\leqq$. For given $p>0$, any polynomial $\prod_{1}^{n}\left(x-\xi_{i}\right)$ satisfying the corresponding condition (1), (2), or (3) is a minimizing polynomial $T_{n}(x)$ for duly chosen weights. (Received December 9, 1952.)

\section{G. G. Murray: Measures in Boolean algebras.}

A theorem of Nakamura and Sunouchi (Proc. Imp. Acad. Tokyo vol. 18 (1942) pp. 333-335) gives a decomposition of a bounded, finitely additive measure defined on a Boolean algebra with respect to a $\sigma$-ideal contained in the algebra, i.e. the measure is split into the sum of two measures, one of which is concentrated in a maximal element of the ideal. By means of Stone's compactification any Boolean algebra $B$ can be represented isomorphically as the collection of open-and-closed sets of a compact Hausdorff space $\Omega$, and a measure $m$ defined on $B$ can be made to correspond to a unique countably additive Baire measure $m^{*}$ in $\Omega$. Taking the Baire sets of the first category in $\Omega$ to be the ideal of the Nakamura-Sunouchi theorem, it is possible to decompose $m$ into the sum of a largest countably additive measure and a purely finitely additive measure. This decomposition was obtained in a different manner by Yosida and Hewitt (Trans. Amer. Math. Soc. vol. 72 (1952) pp. 46-66), but the above method characterizes more precisely the Baire measure corresponding to a purely finitely additive measure since it can be seen to be concentrated in a Baire set of the first category. When the Boolean algebra is taken to be the collection of all subsets of a given set, problems such as the existence of nontrivial, countably additive, two-valued measures and Banach's general measures can be transformed into topological problems in compact Hausdorff spaces. (Received November 17, 1952.) 
201t. Pasquale Porcelli: Concerning uniform completeness of sets of reciprocals of linear functions. II.

This paper is a continuation of the study, begun in an earlier paper of the same title (Bull. Amer. Math. Soc. Abstract 58-6-649), of sets $K:\left\{\left(1+k_{p} x\right)^{-1}\right\}_{p=0}^{\infty}$. To the three characterizations in (I) of sets $K$ which are uniformly complete in $F[0,1]$ is added a fourth, namely: a necessary and sufficient condition for $K$ to be uniformly complete in $F[0,1]$ is that $K$ should be closed in $L^{2}[0,1]$. Furthermore, if $K$ is not closed in $L^{2}[0,1]$, then the closed linear manifold generated by $K$ in $L^{2}[0,1]$ is nowhere dense in $L^{2}[0,1]$. The theorems established in (I) and in this paper concerning $K$ hold also for the sets $K_{n}:\left\{\left(1+k_{p} x\right)^{-n}\right\}_{p=0}^{\infty}$ where $n$ is a positive integer. (Received November 13, 1952.)

\section{2t. M. S. Robertson: Multivalently star-like functions.}

Let $f(z)=a_{1} z+a_{2} z^{2}+\cdots+a_{n} z^{n}+\cdots$ be regular and multivalently star-like of order 2 with respect to the origin for $|z|<1$. A. W. Goodman (Trans. Amer. Math. Soc. vol. 68 (1950) pp. 204-223) has shown that $\left|a_{3}\right| \leqq 5\left|a_{1}\right|+4\left|a_{2}\right|$ when the coefficients are all real. The author, using a different method of approach, shows that, even when the coefficients are complex, the following sharp inequalities hold for all positive integers $n: 6\left|a_{n}\right| \leqq 2 n\left|n^{2}-4\right|\left|a_{1}\right|+n\left(n^{2}-1\right)\left|a_{2}\right|$. Analogous sharp inequalities are obtained for multivalently star-like functions of order $p$ of the form $f(z)$ $=\sum_{p-1}^{\infty} a_{n} z^{n}$ which further substantiate the Goodman conjecture (Trans. Amer. Math. Soc. vol. 63 (1948) pp. 175-192; also vol. 70 (1951) pp. 127-136) for the coeffcients of multivalent functions. (Received November 10, 1952.)

203t. L. B. Robinson: Solution of a system of equations which helps us to compute a complete system of semitensors.

Given the system of equations $-y_{j} \partial f / \partial y_{i}-y_{j}^{\prime} \partial f / \partial y_{i}^{\prime}-y_{j}{ }^{\prime \prime} \partial f / \partial y_{i}{ }^{\prime \prime}-I_{j} \partial f / \partial I_{i}=0$, $i \neq j, i, j=1,2,3,-y_{1} \partial f / \partial y_{1}-y_{1}^{\prime} \partial f / \partial y_{1}^{\prime}-y_{1}^{\prime \prime} \partial f / \partial y_{1}^{\prime \prime}+I_{2} \partial f / \partial I_{2}+I_{3} \partial f / \partial I_{3}=0,-y_{2} \partial f / \partial y_{2}$ $-y_{2}^{\prime} \partial f / \partial y_{2}^{\prime}-y_{2}^{\prime \prime} \partial f / \partial y_{2}^{\prime \prime}+I_{1} \partial f / \partial I_{1}+I_{3} \partial f / \partial I_{3}=0,-y_{3} \partial f / \partial y_{3}-y_{3}^{\prime} \partial f / \partial y_{3}^{\prime}-y_{3}^{\prime \prime} \partial f / \partial y_{3}^{\prime \prime}$ $+I_{1} \partial f / \partial I_{1}+I_{2} \partial f / \partial I_{2}=0$. Write the matrix with first row $y_{1}, y_{1}^{\prime}, y_{1}^{\prime \prime}, I_{1}$, second row $y_{2}, y_{2}^{\prime}, y_{2}^{\prime \prime}, I_{2}$, and third row $y_{3}, y_{3}^{\prime}, y_{3}^{\prime \prime}, I_{3}$. The fundamental solutions of the above system are given by the determinants of the above matrix which involve the $I$. They are three in number. These solutions enable one to compute a complete system of semitensors. (Received October 27, 1952.)

\section{L. V. Robinson: Generalized calculus as related to partial differ-} ential equations.

Basically, the operational and more formal sides of ordinary calculus and ordinary differential equations depend on such operators as $D \equiv d / d x, D_{x} \equiv \partial / \partial x, D_{y}$ $\equiv \partial / \partial y, \cdots$, and on their respective inverses. Hence, to be able to replace these and their inverses with an operator, $\lambda \equiv p_{1} \partial / \partial x_{1}+p_{2} \partial / \partial x_{2}+\cdots+p_{n} \partial / \partial x_{n}$, and its inverse, where the $p_{k}(k=1,2, \cdots, n)$ are understood to be complex-valued functions of of the $x_{k}$ with continuous derivatives in a space $S^{*}$ in which $\left|p_{1}\right| \neq 0$, is then to extend the scope of ordinary calculus. Such an extension may be referred to as a generalized calculus. It is necessary, however, to define the inverse $\lambda^{-1}$ and to establish basic rules of operations with $\lambda$ and $\lambda^{-1}$. Having done this, it is then found that, between these rules and those of ordinary calculus, there is a very close parallelism. To this generalized calculus, it further develops that partial differential equations bear the same relation as ordinary differential do to ordinary calculus. In fact, a one-to-one 
correspondence is found to exist between $\lambda$ and $D$, whence for every ordinary differential equation solvable by any means, an infinite number of partial differential equations which can likewise be solved exists. A means is therefore afforded for greatly increasing the number of types of partial differential equations for which formal solutions can be found; and ordinary linear differential equations correspond only to the class of parabolic linear partial differential equations. (Received November 14, 1952.)

\section{P. C. Rosenbloom: Studies on Maxwell's equations. I.}

Solutions of Maxwell's equations of the form $\exp (i \omega t) F(x, y, z)$ are studied. It is well known that these equations may be reduced to (1) $L Q=\nabla \times Q+\beta Q=A$, where $A$ is a given vector function, $\beta$ is a constant, and $Q=H+i \alpha E$, and $\alpha$ is a suitable constant. It is obvious that if $R$ is a solution of $\Delta R+\beta^{2} R=A$, then $Q=M R=\beta R$ $-\nabla \times R+\beta^{-1} \nabla(\nabla \cdot R)$ is a solution of (1). It is shown conversely that every solution of (1) in a domain $D$ can be expressed in this form, where $R$ is given explicitly in terms of the tangential components of $Q$ on the boundary of $D$. In this way one can give an analogue for (1) of the mean value theorem for harmonic functions and the analogue of spherical harmonics in terms of which all solutions of (1) can be expanded locally. The methods can be applied to quite general systems of equations of elliptic type with constant coefficients. (Received November 17, 1952.)

206t. H. M. Schaerf: Some topological consequences of the existence of measures.

Let $m$ be a $\sigma$-finite countably additive measure on a family $S$ of subsets of a neighborhood space $R$ and $N$ the family of its null sets. Theorem 1: If $R$ is of uniform first countability and $S$ the family of all open sets, then a representation $R=R_{0}+R_{1}$ with $R_{0}$ in $N$ and $R_{1}$ separable exists if and only if $N$ is closed under formation of unions. Theorem 2: If $R$ is a topological group, $m$ is finite, and $S$ and $N$ are left invariant, then either $R$ is locally compact or of the first category or $N$ contains all measurable compact sets. Theorem 3: If $R$ is a metric group of the second category and $m$ is a Borel measure which is positive on all nonvoid open sets, then $m(A) m(B)>0$ implies that $B^{-1} A$ has a nonvoid interior and that every neighborhood of the unit contains an inner point of $A^{-1} A$. (Received December 11, 1952.)

\section{J. T. Schwartz: Abstract boundary values.}

By making use of the fact that every symmetric extension of an operator $T$ is a restriction of its adjoint and by considering the hermitian bilinear form $i\left(T^{*} x, y\right)$ $-i\left(x, T^{*} y\right)$, an alternate approach to the extension theory of symmetric operators can be made. This has the advantage of dealing more directly with the domains of the operators involved than the usual Cayley transform treatment. The method is convenient for the theory of symmetric differential operators, since the abstract and the concrete theory of these operators are brought into adjustment. An account of details will be given in the series of technical reports to be prepared in connection with the ONR spectral theory project at Yale. (Received December 15, 1952.)

\section{8t. J. T. Schwartz: Essential spectra of differential operators.}

The essential spectrum of a self-adjoint operator is the complement in its spectrum of the set of all isolated points of finite multiplicity. A characterization of the essential spectrum is given which yields many properties of this spectrum for self-adjoint operators derived from ordinary differential operators. The essential spectrum is 
independent of boundary conditions, an additive function of the interval on which the differential operator is defined, and positive for formally positive operators. A general criterion can be given which yields many particular cases in which the coefficients can be "slightly" modified without changing the essential spectrum. In this way, $n$th order generalizations of well known results relating the spectrum of a second order linear differential operator to its coefficient functions are obtained. (Received December 22, 1952.)

209t. W. S. Snyder: On derivatives of set functions. Preliminary report.

Let $\Gamma$ be a class of closed subsets of a bounded open set $G$ in the plane, each set $T$ in $\Gamma$ having parameter of regularity greater than a fixed constant $\rho>0$. Let $f$ be a real-valued, non-negative function defined on $\Gamma$, with upper derivative $\bar{D}(f, x)$ defined on a set $A$ of positive measure. Let $S$ be a subset of $A$ with positive measure. We say a constant $M$ is in the upper class if, given $\epsilon>0$, there is a $\delta>0$ such that if $\left\{T_{i}\right\}_{1}^{n}$ are disjoint sets in $\Gamma$ with diam $\left(T_{i}\right)<\delta$ and $\left|\sum_{1}^{n} T_{i}-S\right|+\left|S-\sum_{1}^{n} T_{i}\right|<\delta$, then for somesubfamily $\left\{T_{i_{j}}\right\}_{j=1}^{l}$ with $\left|\sum_{1}^{n} T_{i}-\sum_{j=1}^{l} T_{i_{j}}\right|^{1}<\epsilon$ we have $\sum_{j=1}^{l} f\left(T_{i_{j}}\right)<M$. It is proved that the infimum of numbers $M$ in the upper class is equal to the integral of $\bar{D}(f, x)$ on $S$. (Received November 14, 1952.)

210. C. T. Taam: Non-oscillation and comparison theorems of linear differential equations with complex-valued coefficients.

Consider (A): $W^{\prime \prime}+Q(x) W=0$, where $Q(x)$ is a complex-valued function defined for $x \geqq 0$ and belonging to $L(0, R)$ for every large $R$. Consider only those nontrivial solutions. Let $I$ be an interval which need not be closed or bounded. (A) is said to be disconjugate on $I$ if no solution of (A) has more than one zero on $I$. This criterion is proved: If there exists a real-valued function $m(x)$ AC on every closed interval contained in $I$ and if $m^{\prime}+m^{2}+q_{1}+k q_{2} \leqq 0 \mathrm{AE}$ on $I$ for some $k$, where $q_{1}$ and $q_{2}$ are the real and imaginary parts of $Q$, then (A) is disconjugate on $I$. From this result other explicit criteria are obtained. Among others, the following comparison theorem is given: Let $f(x)$ be real and belonging to $L(0, R)$ for every large $R$, and let $I_{0}$ be an interval which is either closed or open. If $y^{\prime \prime}+f(x) y=0$ is disconjugate on $I_{0}$ and if $f \geqq q_{1}+k q_{2}$ $\mathrm{AE}$ on $I_{0}$ for some $k$, then (A) is disconjugate on $I_{0}$. Application to the complex domain is discussed. (Received November 13, 1952.)

\section{1t. C. T. Taam: On the complex zeros of functions of Sturm- Liouville type.}

Let $Q(z)$ be an analytic function of the complex variable $z$ in a region $D$. Consider only those solutions of $(\mathrm{A}): W^{\prime \prime}+Q(z) W=0$ which are distinct from the trivial solution ( $\equiv 0)$. The following theorem is proved: Let $S$ be a closed region contained in $D$, the boundary $C$ of $S$ being a closed contour. Let $M$ be the maximum value of $|Q(z)|$ on $C$. If $S$ can be divided into $n$ subregions such that each subregion has a diameter not greater than $\pi M^{-1 / 2}$ and that for any two points $z_{1}$ and $z_{2}$ of a subregion the linear segment $z_{1} z_{2}$ lies in $S$, then, when $Q(z)$ is not a constant, each solution of (A) has at most one zero in each subregion and at most $n$ zeros in $S$. The proof of this theorem depends on a previous result of the author (Theorems 5.1, 5.2, Amer. J. Math. vol. 74 (1952) pp. 317-324). Another estimate of the number of zeros in a circle is also given. (Received November 13, 1952.)

212. J. L. Ullman: Eigenvalues of a Toeplitz form. II. 
Let $f(s)$ be a continuous, positive function on $0 \leqq s \leqq 2 \pi$, with Fourier series $\sum_{-\infty}^{\infty} c_{n} z^{n}, z=\exp (i s)$. The form $T_{n}(f, x)=\sum_{0}^{n} \sum_{0}^{n} c_{j-i} x_{i} \bar{x}_{j}$ is the Toeplitz form associated with $f(s)$. It has determinant $T_{n}(f)$, and its eigenvalues, which are real, are $t_{1}^{n}, \cdots, t_{n+1}^{n}$, arranged in ascending order. Let $g(u)$ be a continuous, non-negative function on $0 \leqq u \leqq 1$. The form $T_{n}(f, g, x)=\sum_{0}^{n} \sum_{0}^{n}\left(c_{j-i}+g(i / n) \delta_{i, j}\right) x_{i} \bar{x}_{j}$, with determinant $T_{n}(f, g)$ and eigenvalues $v_{1}^{n}, \cdots, v_{n+1}^{n}$, clearly generalizes $T_{n}(f, x)$. In Bull. Amer. Math. Soc. Abstract 58-6-667, a new proof of the distribution of the $t_{i}^{n}$ was presented. In this paper, that method is extended to find the distribution of the $v_{i}^{n}$. This result yields a proof of a conjecture communicated by Professor Kac that $\left.\lim _{n \rightarrow \infty}\left(T_{n}(f, g)\right)^{1 / n}=\exp \left((1 / 2 \pi) \int_{0}^{1} \int_{1}^{2 \pi} \log f(s)+g(u)\right) d s d u\right)$, a generalization of the formula $\lim _{n \rightarrow \infty}\left(T_{n}(f)\right)^{1 / n}=\exp \left((1 / 2 \pi) \int_{0}^{2 \pi} \log f(t) d t\right)$, due to Szegö. (Received November 13,1952 .)

\section{W. R. Utz: The connectedness of limit sets.}

The principal theorems of the paper are that in a regular continuous flow on a metric space each orbit-closure is a minimal set and that each non-null $\alpha$ or $\omega$-limit set is a minimal set. As a corollary one has that each $\alpha$ and $\omega$-limit set is connected. It is generally known, and easily shown, that in any continuous flow on a compact metric space the $\alpha$ and $\omega$-limit sets are connected. An example is given to show that "compact" cannot be replaced by "complete." Other corollaries to the principal theorems are given and it is pointed out that the theorems hold for a regular discrete flow on a metric space. (Received November 12, 1952.)

\section{H. S. Wall: Hausdorff means with convex mass functions.}

In order for the regular Hausdorff mean $[H, \phi]$ to include the Cesaro mean $(C, 1)$ it is necessary and sufficient that $\phi=\phi_{1}-\phi_{2}+i \phi_{3}-i \phi_{4}$, where $\phi_{j}$ is non-negative, nondecreasing and convex in $[0,1]$. If $L(f)$ denotes $f(x)+x \int_{x}^{1}(1 / t) d f(t)$, then $\phi$ is nonnegative, nondecreasing and convex in $[0,1]$ if and only if $\phi=L(f)$, where $f$ is non-negative and nondecreasing in $[0,1]$. If $n$ is a positive integer, then $[H, \phi]$ $\supset(C, n)$ if and only if $\phi=\phi_{1}-\phi_{2}+i \phi_{3}-i \phi_{4}$, where $\phi_{j}=L^{n}\left(f_{j}\right)$ and $f_{j}$ is non-negative and nondecreasing in $[0,1]$. (Received November 12, 1952.)

\section{Applied Mathematics}

\section{Nachman Aronszajn: Equilibrium deflection of a clamped} rectangular plate.

The deflection for any given load is calculated by using a formula for the projection on $\mathfrak{S}_{1} \oplus \mathfrak{W}_{2}$ in terms of projections on $\mathfrak{W}_{1}$ and $\mathfrak{W}_{2}$ (see N. Aronszajn, Trans. Amer. Math. Soc. vol. 68 (1950) pp. 375-380). This method allows convenient a priori and a postiori evaluations of error. (Received December 16, 1952.)

\section{6t. H. D. Block: A note on contact transformations.}

Many contact transformations: $Q_{i}=f_{i}\left(q_{1}, \cdots, q_{n}, p_{1}, \cdots, p_{n}\right), P_{i}=g_{i}\left(q_{1}, \cdots, q_{n}\right.$ $\left.p_{1}, \cdots, p_{n}\right)$ can be generated by a suitable function $S\left(q_{1}, \cdots, q_{n}, P_{1}, \cdots, P_{n}\right)$ and the equations $Q_{i}=\partial S / \partial P_{i}, p_{i}=\partial S / \partial q_{i}$. It is well known that a given contact transformation can be so generated if and only if the variables $\left(q_{1}, \cdots, q_{n}, P_{1}, \cdots, P_{n}\right)$ form an independent set. Similar results are true for the generating functions $S\left(q_{1}, \cdots, q_{n}, Q_{1}, \cdots, Q_{n}\right), \quad S\left(p_{1}, \cdots, p_{n}, Q_{1}, \cdots, Q_{n}\right)$, and $S\left(p_{1}, \cdots, p_{n}\right.$, $\left.P_{1}, \cdots, P_{n}\right)$. It is a common misconception that every contact transformation can be generated by at least one of these four methods (cf. MacMillan, Theoretical mechan- 
ics: Dynamics of rigid bodies, p. 364 or Goldstein, Classical mechanics, p. 240 and the footnote on p. 248). The purpose of this note is to present examples of contact transformations which can not be generated by any of these four methods. The details will appear in the Journal of Mathematics and Physics. (Received November 13, 1952.)

\section{Y. W. Chen: A mathematical analysis of linearizations of supersonic flow equations.}

This paper is concerned with supersonic flows around the axis of rotational symmetry, viscosity and heat conduction are neglected. Relations between the exact and linearized solutions are investigated for the following initial value problem. Let $\theta$ be the flow angle, $\tan ^{2} \beta=M^{2}-1, M$ being the Mach number. In the neighborhood of a point $P\left(x=x_{0}, y=0\right)$ on the axis of rotational symmetry, the velocity distribution is assigned by $\theta=0, \beta=\beta_{0}+O\left(x_{0}-x\right)^{\gamma}$ for $x_{0}>x$, and $\theta=0, \beta=\beta_{0}+O\left(x-x_{0}\right)^{\delta}$ for $x>x_{0}$. Two types of distributions are studied: type (a) with $\gamma>1 / 2, \delta>1 / 2$, and type (b) with $\gamma>1 / 2, \delta=1 / 2$. For type (a) there is a continuous flow in the neighborhood of $P$ and the customary linearization gives the correct first approximation. For type (b), however, the customary linearization is completely wrong after the leading Mach line $C_{-}$issued from $P$. The streamlines undergo a jump of curvatures in crossing $C_{-}$. In the case of a compression, i.e. $\beta=\beta_{0}+B\left(x-x_{0}\right)^{1 / 2}+O\left(x-x_{0}\right)^{\delta}$ with $\delta>1 / 2, B<0$, the continuous flow breaks down in the neighborhood of the reflected Mach line $C_{+}$issued from $P$, and continuous solution exists only in terms of the characteristic variables. In the case of an expansion, i.e. $B>0$, the flow remains continuous in crossing $C_{+}$, and the space derivatives of $\theta$ and $\beta$ satisfy two Riccati differential equations along the same Mach line $C_{+}$. A correct linearization of type (b) is obtained by making use of the differential equations set up by the author in an early paper published in Communications on Pure, Appl. Math. vol. 5, pp. 52-86. (Received November 12, 1952.)

\section{R. V. Churchill: The operational calculus of Legendre trans- forms.}

The finite linear integral transformation $T\{F(x)\}=\int_{-1}^{1} F(x) P_{n}(x) d x$, where $P_{n}(x)$ are the Legendre polynomials, is the basis of an operational calculus that transforms the differential operator $D\left(1-x^{2}\right) D$ into an algebraic operator. New operational properties of that transformation are presented and illustrated in the solution of boundary value problems. The transforms of several functions are also noted. (Received November 14, 1952.)

\section{9t. Nathaniel Coburn: Some intrinsic relations involving the velocity and vorticity vectors in fluid dynamics.}

Three results involving intrinsic properties of the velocity and vorticity vectors are derived. First, in the case of plane flows, it is well known that the magnitude of the vorticity is equal to the negative of the rate of change of the magnitude of the velocity with respect to displacement in a direction normal to the stream lines plus the magnitude of the velocity times the curvature of the stream lines. It is shown that, for three-dimensional flows, this last term is the component of the vorticity vector along the binormal direction of the stream lines. The components of the vorticity vector along the stream lines and in the direction of the principal normal to these lines are determined. Secondly, it is shown that by use of this result, an intrinsic necessary and sufficient condition for parallel Bernoulli surfaces can be given. Finally, it is shown that if the stream lines are orthogonal to $\infty^{1}$ surfaces, then the rate of 
change of the magnitude of the velocity with respect to a displacement along the stream lines depends upon the mean curvature of these surfaces. (Received October 8, 1952.)

\section{Frank Faulkner: The degenerate problem of Bolza with sepa-} rated end conditions.

The problem treated is that of finding a curve with the following properties: a differential equation $P d x+Q d y+R d z=0$ is to be satisfied along the curve; certain relations of the form $G_{i}\left(x_{0}, y_{0}, z_{0}\right)=0$ and $H_{i}\left(x_{1}, y_{1}, z_{1}\right)=0$ are to be satisfied at the end points of the curve; an expression $F\left(x_{1}, y_{1}, z_{1}\right)-F\left(x_{1}, y_{1}, z_{1}\right)$ is to be maximized; the differential $d x$ is required to be non-negative along the curve. It is shown that there is a unique solution with the desired properties, under certain conditions. In general, there are no solutions if the last restriction is removed. (Received November 13, 1952.)

221. H. E. Fettis: On an integral involved in the theory of high speed scanning antenna.

In a recent paper by Harry Gruenberg (Journal of Applied Physics, July 1952) a formula is derived for the power radiated by a narrow slot of approximately half a wave length, radiating into a parallel plate region. It is the purpose of this paper to indicate how the function $Q(\xi)$ which is involved in the above formula can be expressed in terms of the functions $J_{c}$ and $J_{s}$ defined and tabulated by L. Schwartz (Investigation of some functions related to the Bessel function of zero order, Luftfahrtforschung, February 8, 1944). (Received October 20, 1952.)

222. G. E. Forsythe: Convergence of the conjugate gradient method in Hilbert space. Preliminary report.

Let $A$ be a self-adjoint, bounded, positive-definite operator in a real Hilbert space JC; let the lower, upper bounds of $A$ be $m, M(0<m<M<\infty)$. The conjugate gradient method [M. Hestenes and E. Stiefel, J. Research Nat. Bur. Standards vol. 49 (1952) to appear] is an algorithm for solving an equation $A x=b$, where $b \in \mathcal{H}$. Let $f(x)$ $=(x, A x)-2(x, b)+\left(b, A^{-1} b\right)$. Starting from an arbitrary $x_{0} \in \mathcal{H}$, the points $\left\{x_{n}\right\}$ $(n=1,2, \cdots)$ are chosen so that $x_{n}=x_{0}-\alpha_{0} r_{0}-\cdots-\alpha_{n-1} A^{n-1} r_{0}$, where $r_{0}=b$ $-A x_{0}$, and so that $f\left(x_{n}\right)$ is minimized as a function of the real parameters $\alpha_{i}$. To study the convergence, let $\tau_{n}^{n}=\min _{P_{n}} \max _{\lambda \in S}\left|P_{n}(\lambda)\right|$ over all $n$th degree polyhomials with $P_{n}(0)=1$, where $S C[m, M]$ is the spectrum of $A$; and let $\tau=\tau(S)=\lim _{n} \tau_{n}$. Two new results are easily proved: $\operatorname{I}$. $\lim \sup _{n}\left[f\left(x_{n}\right) / f\left(x_{0}\right)\right]^{1 / 2 n} \leqq \tau$. II. Let $\int_{S} \lambda d e_{\lambda}$ be the spectral decomposition of $A$. Suppose $S=\bigcup_{i=1}^{r} I_{i}$, where each $I_{i}$ is a closed interval, and for each $i$ suppose that $\lambda, \mu \in I_{i}$ implies $\left|\left(e_{\lambda} r_{0}, r_{0}\right)-\left(e_{\mu} r_{0}, r_{0}\right)\right| \geqq \epsilon|\lambda-\mu|, \epsilon>0$. Then $\lim _{n}\left[f\left(x_{n}\right) / f\left(x_{0}\right)\right]^{1 / 2 n}=\tau$. Previously known convergence estimates of W. Karush and of R. M. Hayes follow from I and from known connections between $\tau$ and the transfinite diameter of $S$. (Received November 12,1952.)

\section{H. E. Goheen: On a nonlinear boundary value problem.}

The author establishes the existence and uniqueness of the boundary value problem, $\phi(0)=a_{0}<1, \phi(\infty)=0$, for the ordinary differential equation $\phi^{3} \phi^{\prime \prime}+2 \phi^{2} \phi^{\prime 2}+v \phi^{\prime}$ $=0$. (Received November 6, 1952.)

224. Michael Goldberg: Rotors within rotors. 
The curve of Ribaucour can rotate continuously within an equilateral triangle while remaining tangent to its three sides. A convex involute of the deltoid can rotate continuously within a square while remaining tangent to its four sides. It is shown that the curve of Ribaucour can be inscribed in the deltoid involute and turned continuously in a constrained manner through all orientations. These curves have technical application in the theory of testing for roundness. (Received November 6, 1952.)

\section{L. E. Payne: An equality involving virtual mass and polariza-} tion.

Let the symbols $W_{i j}$ and $P_{i j}$ denote the components of the virtual mass and polarization tensors respectively (see Schiffer and Szegö, Trans. Amer. Math. Soc. vol. 67, pp. 130-205). In the case of an axially symmetric body let the subscript 1 refer to the direction parallel to the axis of symmetry and the subscript 2 to a direction perpendicular to the axis of symmetry. For an axially symmetric body with a simplyconnected meridian section the following relationship can be shown to exist: $2 W_{11}$ $=P_{22}-V$. From this equality follow a number of important inequalities: (a) $P_{11}+2 P_{22}$ $\geqq 6 V$; (b) $W_{11}+W_{22} \geqq 3 V / 2$, etc. The existence of the relationship between $W_{11}$ and $P_{22}$ was observed in certain special cases by Schiffer and Szegö but no proof was given. (Received November 10, 1952.)

\section{Charles Saltzer: An abridged block method for the solution of} the Laplace difference equation.

A procedure for solving the boundary value problem is developed which is analogous to Schwartz's alternating procedure for the solution of the Laplace equation. The use of this method is based on the known formula for the solution of the difference equation for a rectangular net, and the convergence of the iteration is proved. (Received November 12, 1952.)

\section{J. B. Serrin, Jr.: Some comparison theorems for subsonic flow} of gases.

Let $F$ and $\bar{F}$ be irrotational flows (both plane or both axi-symmetric) of an incompressible ideal fluid, uniform at infinity, and bounded, respectively, by streamlines $S$ and $\bar{S}$. If $S$ and $\bar{S}$ have a common arc $M N$, while to the right of $M N, \bar{S}$ is above $S$, and to the left of $M N, \bar{S}$ is below $S$, then the following inequality persists: $q(M) / q(N) \leqq \bar{q}(M) / \bar{q}(N)$, where $q(P), \bar{q}(P)$ denote respective flow speeds at a point $P$ (Journal of Rational Mechanics vol. 1 (1952) p. 563). A similar inequality is shown to hold for the following cases of subsonic flow of a compressible medium; (1) when there is a point $P$ on $M N$ where $q(P)=\bar{q}(P)$; (2) when either flow is uniform; (3) when both flows are given by the linearized equations. These results imply explicit inequalities for the velocity gradient along straight arcs and at inflection points of a streamline. Similar conclusions are obtained for flows taking place in channels. (Received November 10, 1952.)

\section{8t. D. V. Widder: Positive temperatures on a semi-infinite rod.}

In 1944 the author proved that the only non-negative solution $u(x, t)$ of the heat equation $u_{x x}=u_{t}$ which vanishes as $t$ approaches $0+, u(x, 0+)=0,-\infty<x<\infty$, is the identically vanishing one. An analogous result is proved here for the semi-infinite rod. That is, if a solution $u(x, t)$ is non-negative for $0<x<\infty, 0<t<\infty$, and if $\lim u(x, t)$ is 0 as $(x, t)$ approaches (two-dimensionally) each boundary point of that quadrant, then $u(x, t)$ is identically zero in the quadrant. Also an integral representa- 
tion is obtained for every solution non-negative in the quadrant. (Received October 10, 1952.)

\section{Geometry}

\section{Iacopo Barsotti: Equivalence theories on algebraic varieties and} the definition of the Picard variety. Preliminary report.

Let $V$ be an $n$-dimensional algebraic variety over a field $k$ (usually algebraically closed). The customary definitions and properties of continuous (or algebraic) equivalence between the integral cycles of $V$ are established by algebraic means. It is proved that, under slightly restrictive conditions, the elements of an irreducible $m$-dimensional algebraic system $\mathfrak{E}$ of $n$-1-dimensional cycles cut on a generic irreducible cycle of $\mathbb{E}$ a linear system of dimension $m-1$ (the characteristic system). It is also proved that the cycles of $\mathfrak{E}$ are all linearly equivalent to each other if either (1) $\mathfrak{E}$ is unirational, or (2) $\mathbb{E}$ has index 1 and dimension $>1$. It is proved that if $\mathbb{E}$ is maximal, and $G$ is the representative variety of $\mathscr{E}$, then $G$ is birationally equivalent to the product of a variety $F$ and a projective space of dimension equal to the dimension of the largest linear system containing the general element of $\sqrt{ }$. By induction on the dimension of $V$ it is proved that, for a given $V$, the dimension of $F$ has a maximum $q$ (the irregularity of $V$ ), and that all the $F$ 's having dimension $q$ are birationally equivalent to each other. It follows that if $F$ is any of these, it is an abelian variety (the Picard variety of $V$ ), and that $F$ is, as a group, isomorphic to the factor group of the group of $n$-1-dimensional cycles of $V$ linearly equivalent to 0 , in the group of those continuously equivalent to 0 . The proofs are of an algebraic nature, and rest heavily on the recent work by the author on algebraic correspondences. (Received November 3, 1952.)

230. Eugenio Calabi: On tangent bundles, holonomy groups, and torsion. Preliminary report.

Let $M^{n}$ be a compact, differentiable, $n$-dimensional, affinely connected manifold. The associated holonomy group with respect to a point $p \in M^{n}$, i.e. the group of linear transformations of the tangent space at $p$ obtained from parallel displacements along rectifiable loops originating at $p$, is a subgroup of $G L(n, R)$, whose closure we call $H$; then the tangent bundle of $M^{n}$ admits $H$ as structural group. Conversely, if the tangent bundle admits a subgroup $H$ of $G L(n, R)$ as structural group, then there exists an affine connection, but not generally a symmetric one, in $M^{n}$, whose holonomy group is contained in $H$. The existence of a symmetric affine connection in $M^{n}$ with holonomy group contained in $H$ is considered as a general problem, whose applications include the following important cases. Let $n=2 k ; M^{n}$ is an almost-complex manifold if the structural group of the tangent bundle is isomorphic to a subgroup of any one of the following: (a) $G L(k, C)$, (b) $S p(2 k, R)$, or (c) $U(k)$. Theorem. If $M^{2 k}$ is an almost-complex manifold, then there exists a symmetric affine connection with holonomy group contained in the above named groups respectively, if and only if $M^{2 k}$ is: (a) a complex manifold, (b) a symplectic manifold, or (c) a Kaehler manifold. (Received November 14, 1952.)

\section{N. A. Court: Pascal's theorem in space.}

The following two propositions may be considered as extensions of Pascal's theorem to three-dimensional Euclidean space. I. The four triads of coterminous edges of a tetrahedron $(T)$ are cut, respectively, by four planes in four triads of points lying on the same quadric surface if, and only if, the four secant planes meet the faces of $(T)$ 
opposite the respective vertices in four lines which are (i) coplanar or (ii) hyperbolic. II. If the three pairs of opposite edges of a tetrahedron $(T)$ taken two-by-two are cut, respectively, by three planes, the six pairs of points of intersection lie on a quadric surface if, and only if, the three lines determined in the secant planes by the traces of the respective third pairs of opposite edges of $(T)$ are coplanar. (Received November $4,1952$.

\section{2t. John DeCicco: Extension of Kasner's inverse of Meusnier's theorem to Riemannian space.}

In a certain region of a Riemannian space $V_{n}$, consider a unit vector $\mu_{i}(x ; \dot{x})$ whose components possess suitable differentiability conditions relative to the lineal elements $(x ; \dot{x})$ in the region, and which are homogeneous of degree zero in the $\dot{x}$. A system $S$ of curves in this region of $V_{n}$ is said to have the Meusnier property relative to this vector $\mu_{i}(x ; \dot{x})$ if every curve $C$ of $S$ through the point $x$ in the direction $\dot{x}$ is orthogonal to the vector $\mu_{i}(x ; \dot{x})$, and if its radius of geodesic curvature $\rho$ at this lineal element $(x ; \dot{x})$ varies directly as the cosine of the angle $\theta$ between its principal normal and the fixed direction $\mu_{i}(x: \dot{x})$. Thus $\rho=R \cos \theta$, where $R=R(x ; \dot{x})$, is homogeneous of degree zero in the $\dot{x}$. Such a system $S$ is defined by the differential equation $\mu_{i} \ddot{x}+\nu$ $=2$, where $\nu=\nu(x ; \dot{x})$ is homogeneous of degree two in the $\dot{x}$. The auxiliary condition $\mu_{i} \dot{x}^{i}=0$ must be satisfied. Systems $S$ of this type are invariant under the group of arbitrary point transformations. For the classical three-dimensional case, see Kasner, The inverse of Meusnier's theorem, Bull. Amer. Math. Soc. vol. 14 (1908) pp. 461-465. (Received November 12,1952.)

\section{Harley Flanders: Development of an extended exterior differ- ential calculus.}

The purpose of this paper is to set up an algebraic machinery for the theory of manifolds with affine connections. Tensor spaces of $p$-vectors with $q$-form coefficients are introduced and their algebraic relations studied. An affine connection on a manifold $M$ is defined as a certain type of linear operator on the space of vector fields to the space of vectors with one form coefficients. It is proved that such an operator induces further operators on each space of $p$ vector fields with $q$ form coefficients to the space of $p$ vectors with $(q+1)$ form coefficients. Application is made to the systematic derivation of identities between the displacement vector, connection forms, torsion tensor, etc. Also further application is made to the Chern curvature integra in the case of Riemannian manifolds. (Received November 5, 1952.)

\section{4t. P. C. Hammer: Class-equivalence of functions and curves. I.} Preliminary report.

Let $f(x)$ and $g(x)$ be two real-valued functions defined on an open interval $(a, b)$. If for every null sequence of nonvanishing terms, $h_{n}$, such that $\left[f\left(x+h_{n}\right)-f(x)\right] / h_{n}$ has a unique limit in the compactified real number system, the sequence $\left[f\left(x+h_{n}\right)\right.$ $\left.-f(x)-g\left(x+h_{n}\right)+g(x)\right] / h_{n}$ has the limit zero, then $f$ and $g$ are said to be classequivalent at $x$. A necessary and sufficient condition that $f$ and $g$ be class-equivalent for all $x$ in $(a, b)$ is that they differ by a constant. Extensions of this result are made to vector-valued functions defined on a linear space and to curves and surfaces. The theory provides a new tool for the establishment of uniqueness of solution curves and surfaces when Lipschitz type conditions do not prevail. (Received December 4, 1952.) 
235. P. C. Hammer: Inversion of a planar symmetrization process.

Let $C$ be a planar convex body (a closed bounded convex set with positive area). The convex body $B$, obtained by translating diameters of $C$ so that their midpoints fall on a fixed point, is called the symmetroid of $C$ and the process is called point symmetrization or central symmetrization. The symmetroid of $C$ has the same breadth as $C$ in each direction. Given a point symmetric planar convex body $B$, a construction of all convex bodies which have $B$ as a symmetroid is given, thus completely inverting point symmetrization process. For a given point symmetric convex body $B$ and an outwardly simple line family $F$ there exists a one-parameter family of convex bodies with a common subfamily of diameters which extend to give $F$ and such that the symmetroid of each such convex body is similar to $B$. (Received September 29,1952.)

236t. Edward Kasner and John DeCicco: The special physical systems in Riemannian space of dynamical trajectories, catenaries, brachistochrones, and velocity curves.

A physical system $S_{k}$ of $\infty^{2 n-1}$ curves in a Riemannian space $V_{n}$ is defined as follows. For an arbitrary field of force in $V_{n}$, a curve $C$ is in $S_{k}$ if a constrained motion along $C$ is possible such that the osculating geodesic surface at each point $x$ of $C$ possesses the force vector at $x$ as a tangent, and the pressure $P$ along the principal normal to $C$ at $x$ is proportional to the principal normal component $N$ of the force vector. Thus $P=k N$ where $k$ is the constant factor of proportionality. Starting from fundamental physical principles, it is proved that dynamical trajectories, general catenaries, brachistochrones, velocity systems are all special systems $S_{k}$ for $k=0,1$, $-2, \infty$, respectively. Also every natural family is a special velocity system. In a conservative field of force, any system $S_{k}$ is a natural family and the associated calculus of variations problem is discussed. (Received November 13,1952.)

\section{Paolo Lanzano: Tensorial connections.}

Tensorial connections were introduced by Bompiani (Rendiconti Lincei (VIII) vol. I) : the purpose of this paper is to generalize them for any tensor field. Given a tensor field $A(x)(p ; q)$, ( $p$ indices of contravariance, $q$ of covariance) defined on $V_{n}$, it is asked that the combinations $\nabla_{t} A=\partial_{t} A+\sum L A$ be tensors $(p ; q+I)$ (tensor derivatives); the $L$ 's, functions of $V_{n}$ with $2(p+q)+I$ indices, are coefficients of a tensorial connection: its transformation law is established. Coincidence of covariant and tensor derivatives is taken as basis for equivalence of tensorial and vectorial (affine) connections: it is proved that the vanishing of a tensor $(p+q ; p+q+I)$ is the necessary and sufficient condition for the equivalence of a given tensorial and vectorial connection. The integrability conditions of the transformation law of the $L$ 's give rise to a curvature tensor $(p+q ; p+q+2)$. Second order tensor derivatives can be obtained by use of the $L$ and another connection $M$ with $2(p+q+I)+I$ indices: the necessary and sufficient condition for them to be independent of the order of derivation is the vanishing of the curvature tensor of $L$ and of a new tensor, the torsion of $L$ and $M$. (Received November 5,1952 .)

\section{T. Minagawa and Tibor Radó: On the rigidity of the torus.}

Let $B_{o}, B_{i}$ denote the outer belt (of positive curvature) and the inner belt (of negative curvature) of the torus $T$, and let $z$ be a vector function on $T$, of class $C^{\prime}$, which induces an infinitesimal deformation of $T$. By a theorem of Rembs (extended to the class $C^{\prime}$ case by the authors), the infinitesimal deformation is trivial on $B_{0}$. Thus on the boundary of $B_{i}$, which is of negative curvature in its interior, the infinitesimal 
deformation coincides with a trivial one. By an extension of one of the authors' previous results concerning the rigidity of surfaces of negative curvature, it is inferred that $z$ is trivial on $B_{i}$ also. It follows that the torus is infinitesimally rigid. (Received October 8, 1952.)

\section{9t. J. T. Schwartz: DeRham's theorem for arbitrary spaces.}

By considering pairs $(S, T)$ of compact subspaces of a Euclidean space $E^{N}$ and appropriate local spaces of forms near $S$ and $T$, a theory of differential forms on arbitrary finite-dimensional compact spaces can be constructed. The construction leads to groups $H_{n}(S, T)$ which are shown to satisfy all the Eilenberg-Steenrod cohomology axioms for cohomology with real coefficients. In the case where $S$ is a $C^{\infty}$ manifold regularly imbedded in $E^{N}$, the groups $H_{n}(S, T)$ can easily be seen to depend only on the space of forms on $S$. In this way, the abstract method of Eilenberg-Steenrod yields deRham's theorem for an arbitrary compact manifold. Since dualization for groups derived from forms is especially simple, this method also gives elementary proofs of the Jordan curve theorem and other theorems of the Alexander type. (Received December 8, 1952.)

\section{Oswald Wyler: Perpendicularity in absolute geometry.}

Perpendicularity of lines and planes in a space satisfying Hilbert's axioms of incidence may be characterized by three axioms which, like the axioms of incidence, are satisfied in any open set of a Euclidean, hyperbolic, or elliptic geometry. If a space satisfying these axioms is embedded into a projective geometry by the construction of bundles, perpendicularity becomes part of a well-determined "absolute" polarity of the projective geometry. This polarity is degenerate if, and only if, two distinct proper planes have the same bundle of perpendicular lines. The theory is easily extended to spaces of any finite dimension $\geqq 3$. No notions of order are needed. (Received November 12, 1952.)

\section{Logic AND Foundations}

241. Hermann Rubin and P. C. Suppes: Transformations of systems of relativistic particle mechanics.

A set of axioms is given for systems of relativistic particle mechanics (in the sense of the special theory of relativity). Transformation theorems are then established analogous to the theorems established by McKinsey and Suppes for classical particle mechanics (Transformations of systems of classical particle mechanics, forthcoming in Journal of Rational Mechanics and Analysis). (Received November 14, 1952.)

\section{2t. A. R. Schweitzer: On the foundations of geometry.}

The foundations of geometry are classified into four types: descriptive, metrical, projective and group-theoretic. With each type topological concepts are associated. Common to the first three types are reflexiveness, symmetry, and transitiveness of the corresponding (undefined) generating relations. The latter are symbolized by $K, E, K^{*}$ expressing respectively sameness of sense, equality (leading to congruence), and cospatiality of $(n+1)$-ads of points $(n=1,2,3, \cdots)$. The relations $E$ and $K^{*}$ are respectively termed metrical and projective analogues of the relation $K$. Correspondingly, axiomatic systems generated by the relations $E$ and $K^{*}$ are metrical and projective analogues of the system generated by the relation $K$. Reference is 
made to the author's theory of geometrical relations, Amer. J. Math. vol. 31 (1909) p. 366. (Received November 12, 1952.)

243t. A. R. Schweitzer: On the transition from Grassmann's space analysis to combinatorial topology.

It is assumed that the simplex is a fundamental concept of the foundations of geometry, Grassmann's space analysis, and combinatorial topology. Genetically, topology involves both generalization and specialization. Subjects discussed include: 1. The simplex. 2. Sets of simplexes. 3. Homology group. 4. Correspondence. 5. Invariance. 6. Fundamental group. (Received November 12, 1952.)

\section{4t. A. R. Schweitzer: Topological problems in the foundations of geometry.}

In this paper topological concepts are associated with descriptive geometry. The author discusses assumptions under which the following statements are valid. I. Given a finite set of points $S$ in a descriptive plane $P$, there exists in $P$ a triangle $T$ such that $S$ lies "entirely within" $T$. In particular, $S$ may consist of the vertices of two triangles $T_{1}$ and $T_{2}$. If the triangle $T_{1}$ lies entirely within the triangle $T$, then $T$ can be subdivided into a set of triangles, including $T_{1}$, such that $T_{1}$ and $T$ have the same or opposite senses. II. Given the triangles $T_{1}$ and $T_{2}$ in $P$, there exists in $P$ a triangle $T$ such that $T$ lies "entirely without" $T_{1}$ and $T_{2}$. Two triangles in $P$ of which one lies entirely without the other can be joined by a sequence of "consecutive" triangles in $P$ and therefore have the same or opposite senses. (Two triangles in $P$ are consecutive if they have two vertices and corresponding sides in common and the remaining vertices are opposite relatively to the common side.) The preceding is generalized to $n$ dimensions $(n=2,3, \cdots)$. (Received November 12, 1952.)

\section{5t. D. E. van Tijn: An interpretation of free variables.}

Given a formal system $L$ it is possible to define another system $L^{\prime}$ with the following properties: (1) If $x$ is a variable in $L, x^{*}$ will be a constant in $L^{\prime}$. (2) There are variables $t$ in $L^{\prime}$, and $x^{*}(t)$ is an individual term. One can then map formulas of $L$ containing free variables into closed formulas in $L^{\prime}$ and variables of $L$ into constants of $L^{\prime}$, and this mapping will have all the properties which are accepted as giving "meaning" to closed formulas. The resultant system $L^{\prime}$ has application in describing restricted quantification and such concepts as "a variable depends on another." (Received November 21, 1952.)

\section{Statistics AND Probability}

\section{P. R. Rider: The distribution of the product of ranges in samples} from a rectangular population.

The distribution of the product of ranges of independent, random samples from a continuous rectangular population is considered. For the distribution of the product of two samples a general formula is given. This formula fails to hold if the sample sizes are the same or if they differ by unity. Special consideration is given to these cases. The distribution of the product of $k$ samples of equal size is derived. Simple formulas for the moments about the origin are given for all of these distributions. (Received October 14, 1952.) 
247t. F. L. Spitzer: A limit theorem for two-dimensional brownian motion.

Let $X_{i}(t)$ be independent Wiener processes with $X_{i}(0)=0, i=1,2, \cdots, n$. Then $R_{n}(t)=\left(X_{1}^{2}(t)+\cdots+X_{n}^{2}(t)\right)^{1 / 2}$ denotes the distance from the origin at time $t$ of a particle in brownian motion in an $n$-dimensional euclidean space. For $n \geqq 3$, it was shown by Dvoretzky and Erdös (Proceedings of the 2nd Berkeley Symposium, University of California Press, 1951) that for monotone decreasing functions $\phi(t)$ the probability $P_{n}=\operatorname{Pr}\left\{R_{n}(t)<t^{1 / 2} \phi(t)\right.$ for arbitrarily large $\left.t\right\}$ equals zero or one according as the infinite series $\sum_{k=1}^{\infty} \phi^{n-2}\left(2^{k}\right)$ converges or diverges. The result of the present paper is the analogous theorem for the two-dimensional case $(n=2)$ : With the above notation, $P_{2}$ equals zero or one according as the infinite series $\sum_{k=1}^{\infty}\left[\log \phi\left(2^{k}\right)\right]^{-1}$ converges or diverges. The method which is used to obtain this result also yields that of Dvoretzky and Erdös. (Received October 17, 1952.)

\section{8t. F. L. Spitzer: The angular component of two-dimensional brownian motion.}

Let $X(t)$ and $Y(t)$ be independent Wiener processes defining a stochastic vector process $Z(t)=X(t)+i Y(t)$ of two-dimensional brownian motion. Let $R(t)=|Z(t)|$ and $\theta(t)=\operatorname{amp} Z(t) \cdot \theta(t)$ ranges over the real numbers, so that it may be interperted as the angular component of brownian motion on the Riemann surface of $\log z . \theta(t)$ becomes a well-defined stochastic process if the initial conditions $\theta(0)=0$ and $R(0)$ $=R \neq 0$ are imposed. Then $\theta(t)$ is a continuous function of $t$, with probability one, during any finite interval $\left[t_{1}, t_{2}\right]$. By solving the diffusion equation on the Riemann surface of $\log z$, explicit formulae are obtained for the distribution functions of $\theta(t)$. The distributions of transition and of one-sided absorption satisfy the relation $G(\alpha, t ; R)=\operatorname{Pr}\{\theta(t)<\alpha \mid \theta(0)=0, R(0)=R\}=1-2^{-1} \operatorname{Pr}\left\{\max _{0 \leqq \tau \leqq t} \theta(\tau)>\alpha \mid \theta(0)=0\right.$, $R(0)=R\}$, and $\partial G(\alpha, t ; R) / \partial \alpha$ is a symmetric density in $\alpha$ with infinite absolute mean. With proper normalization, simple asymptotic distributions are shown to exist for $\theta(t)$, viz. $\lim _{t \rightarrow 0} G\left(\alpha t^{1 / 2} / R, t ; R\right)=\left(1 /(2 \pi)^{1 / 2}\right) \int_{-\infty}^{\alpha} e^{-s^{2} / 2} d s$ and $\lim _{t \rightarrow \infty} G\left(\alpha \log t^{1 / 2}, t ; R\right)$ $=(1 / \pi) \int_{-\infty}^{\alpha} d s /\left(1+s^{2}\right)$. (Received October 17, 1952.)

\section{TOPOLOGY}

\section{R. D. Anderson: A homogeneous one-dimensional compact con-} tinuous curve.

Let $M$ denote the Sierpinski universal curve (a one-dimensional analogue of the Cantor middle-third set in Euclidean three space), let $A_{1}, \cdots, A_{k}$ denote $k$ points of $M$, and let $B_{1}, \cdots, B_{k}$ denote $k$ points of $M$; then there exists a homeomorphism $f$ of $M$ onto itself with for each $i, 1 \leqq i \leqq k, f\left(A_{i}\right)=B_{i}$. (Received November 14,1952.)

\section{B. J. Ball: Some theorems concerning spirals in the plane.}

In September, 1948, R. L. Moore delivered an address on the subject Spirals before a meeting of Section A of the American Association for the Advancement of Science held in conjunction with the summer meetings of the American Mathematical Society and the Mathematical Association of America. Using definitions introduced by Professor Moore, it is shown that there exists a closed, bounded, totally disconnected point set $M$ in the plane such that every arc containing it spirals down on some point. In order that $M$ should be a subset of a nonspiraling arc, it is necessary and sufficient that it be a subset of the sum of the elements of a continuous and 
equicontinuous collection $G$ of mutually exclusive nonspiraling arcs such that $G$ with respect to its elements as points is closed, bounded, and totally disconnected. Certain related theorems are also established. (Received November 13, 1952.)

\section{R. H. Bing: Locally tame surfaces are tame.}

A set $M$ is tame in a geometric complex $K$ if there is a homeomorphism of $K$ onto itself that carries $M$ onto a subcomplex of some simplicial subdivision of $K$. $M$ is locally tame in $K$ if for each point $p$ of $M$ there is a neighborhood $N$ of $p$ in $K$ and a homeomorphism $f$ of $\bar{N}$ onto a geometric complex that carries $M \cdot \bar{N}$ onto a subcomplex of $f(\bar{N})$. If $M$ is a locally tame closed subset of a triangulated 3-manifold $K$ such that no arc contains an open subset of $M$, then $M$ is tame in $K$. Consequently any 2-manifold with or without boundary which is locally tame in $E^{3}$ is tame there. Also, any 3-manifold with boundary can be triangulated. (Received November 13, 1952.)

\section{Raoul Bott and Hans Samelson: On the Pontryagin product.}

The space $E$ of all paths in a space $X$, starting at a fixed point $x_{0}$, and its subspace $\Omega$ of closed paths permit a composition $\Omega \times E \rightarrow E$ (a loop followed by a path is a path). From this one can define an operation of the homology group $H(\Omega)$ on the groups $E_{r}(r \geqq 2)$ of the spectral sequence of $E$, which commutes with the differentials $\phi_{r}$. Using this one shows that the Pontryagin ring of $\Omega$, when $X$ is union of $k$ spheres, of dimensions $n_{i}$, with a point in common, is the free associative algebra generated by $k$ elements of dimensions $n_{i}-1$; actually a more general theorem is proved. (Received October 31, 1952.)

253t. R. E. Chamberlin and J. H. Wolfe: Homeomorphisms of differentiable manifolds. Preliminary report.

Let $M^{n}$ be a differentiable $n$-manifold and let $f$ be a homeomorphism of $M^{n}$ onto itself. Given $p \in M^{n}$, let $g_{1}, g_{2}: E^{n} \rightarrow M^{n}$ be differentiable homeomorphisms of $E^{n}$ into $M^{n}$ with $p \in g_{1}\left(E^{n}\right)$ and $f(p) \in g_{2}\left(E^{n}\right)$. ( $E^{n}$ is euclidean $n$-space with a fixed coordinate system.) Put $h=g_{2}^{-1} f g_{1}$ and let $h_{\epsilon}(q)=\lambda(\epsilon) \int_{\delta(\epsilon)} h d v$. Here $\lambda(\epsilon)$ is the reciprocal of the volume of the solid $n$-sphere of radius $\epsilon$ and the integral is taken over the solid $n$-sphere of radius $\epsilon$ with center at $q$. A point $p \in M^{n}$ is called a regular point for $\left(f, g_{1}, g_{2}\right)$ if there exist $\delta_{1}>0, \delta_{2}>0$ such that $h_{\epsilon}(q)$ is a homeomorphism in the $\delta_{1}$-nbd. of $g_{1}^{-1}(p)$ for every $\epsilon<\delta_{2}$. It is shown that if $p$ is regular for $\left(f, g_{1}, g_{2}\right)$, then it is regular for $\left(f, g_{1}^{\prime}, g_{2}^{\prime}\right)$ where $g_{1}^{\prime}$ and $g_{2}^{\prime}$ are differentiable homeomorphisms of $E^{n}$ into $M^{n}$ such that $g_{1}^{-1} g_{1}^{\prime}$ and $g_{2}^{-1} g_{2}^{\prime}$ are regular homeomorphisms of open subsets of $E$ containing $\left(g_{1}^{\prime}\right)^{-1}(p)$ and $\left(g_{2}^{\prime}\right)^{-1}(f(p))$ respectively. (Reg. hom. means differentiable with nonvanishing jacobian.) Let $A_{f}$ be the subset of $M$ consisting of those points which are not regular. If $f$ is lipschitz, it is shown that $\operatorname{dim} A_{f} \leqq n-2$ (lipschitz relative to a given differentiable metric). Other properties of $A_{f}$ are also determined. (Received November 13, 1952.)

254. Eldon Dyer: Concerning irreducibility of the sum of the elements of a continuous collection of continua.

In 1935, B. Knaster showed (Fund. Math. vol. 25 (1935) pp. 568-577) that there exists a continuous collection $G$ of mutually exclusive nondegenerate continua such that $G$ is an arc with respect to its elements and $G^{*}$ (the sum of all of the elements of $G$ ) is a compact irreducible metric continuum. He raised the question whether there 
exists a collection fulfilling these conditions together with the additional condition that all of its elements are topologically equivalent to each other. It is shown in the present paper that there does not exist a continuous collection $G$ of mutually exclusive, nondegenerate, decomposable, continua such that $G^{*}$ is a compact irreducible metric continuum. This is an extension of results of E. E. Moise (Bull. Amer. Math. Soc. vol. 55 (1949) pp. 810-811) and M. Hamstrom (Bull. Amer. Math. Soc. vol. 58 (1952) p. 204). (Received December 5, 1952.)

255. D. O. Ellis and H. D. Sprinkle: Topology in B-metrized spaces.

In a preliminary announcement (David Ellis and H. D. Sprinkle, Bull. Amer. Math. Soc. vol. 58 (1952) pp. 573-574) the Boolean metrization of zero-dimensional spaces and some other results were announced. In addition, it may be shown that: (1) The tier function of a B-metrized space is normal (for terminology here and below see Vaidyanathaswami, Treatise on set topology, Indian Math. Soc., 1947); (2) Every $B$-metrized space is completely regular; (3) The distance function of a B-metrized space is continuous simultaneously; (4) Every B-metrized space has a completion of the CantorHausdorff-Meray type; (5) There exist Boolean metrizable weak product topologies on cartesian products of Boolean metrized spaces. (Received November 13, 1952.)

\section{J. S. Griffin, Jr.: $A$ condition that a fibre space be an E-F} bundle.

It is proved that, if $\left\{X, B, \pi, \Omega, \phi_{U}\right\}$ is a fibre space in the sense of S. T. Hu (Proc. Amer. Math. Soc. vol. 1 (1950) pp. 756-762) satisfying the conditions (1) for any points $x$ and $y, \pi^{-1}(x)$ and $\pi^{-1}(y)$ are homeomorphic, (2) for any member $U$ of $\Omega$ and any $a, b$, and $x$ such that $a \in U$ and $b \in U$ and $x \in \pi^{-1}(a), \phi_{U}\left(a, \phi_{U}(b, x)\right)$ $=x$, then there is an $E-F$ bundle (Steenrod, The topology of fibre bundles, pp. 18-20) with bundle space $X$, base space $B$, and projection $\pi$; conversely, if $X, B$, and $\pi$ are respectively the bundle space, base space, and projection of an $E-F$ bundle, then there are $\Omega$ and $\phi_{U}$ such that $\left\{X, B, \pi, \phi_{U}\right\}$ is a fibre space satisfying these conditions. There are also slightly weaker, though considerably less attractive, conditions than those given. This result may be used to show, for example, that every fibre space with totally disconnected fibre and connected locally arcwise connected base space is an $E-F$ bundle. (Received October 31, 1952.)

\section{7t. Mary-Elizabeth Hamstrom: Concerning certain types of webs.}

In the author's dissertation (Bull. Amer. Math. Soc. Abstract 58-6-715, to appear in Trans. Amer. Math. Soc.) a $W_{n}$ set was defined as follows: If $n>1$, a $W_{n}$ set is a compact continuum $M$ for which there exists a family $F$ of $n$ elements such that (1) each element of $F$ is an upper semicontinuous collection of mutually exclusive continua which fills up $M$ and is an arc with respect to its elements and (2) if $G$ is a collection of continua each belonging to some, but no two to the same, collection of the family $F$, then the continua of the collection $G$ have a point in common and their common part is totally disconnected. In that paper it was stated without proof (Theorems 13 and 14) that in the plane there exists a $W_{3}$ set $M$ whose boundary is the point set $B(M)$ and which has a complementary domain whose boundary, $J$, contains six limit points of $B(M)-J$, but that no $W_{3}$ set has a complementary domain whose boundary contains seven such points. It is the purpose of this paper to prove these theorems. (Received November 13, 1952.) 
258. Frank Harary and G. E. Uhlenbeck: On the number of free Husimi trees.

A "Husimi tree" is a connected graph in which no line lies on more than one cycle. A "pure" Husimi tree contains only one type of polygons; a "mixed" Husimi tree is not pure. We have extended Otter's procedure for trees (Ann. of Math. vol. 49 (1948) pp. 583-599) to the counting of the topologically different free Husimi trees in terms of the rooted ones (Bull. Amer. Math. Soc. Abstract 58-2-131). This extension makes use of the notion of the "dissimilarity characteristic" of a Husimi tree (Harary and Norman, Ann. of Math., to appear). Explicit formulas will be presented for pure Husimi trees consisting of triangles ("cacti"), rectangles, pentagons, and hexagons. The counting problems for mixed Husimi trees can be reduced to the case of pure ones. (Received November 13, 1952.)

\section{Edwin Hewitt: Representation of functions as Fourier-} Stieltjes transforms.

Let $G$ be a locally compact Abelian group and let $G^{*}$ be the group of continuous characters of $G$. Let $(x, y)$ denote the value of the character $y \in G^{*}$ at the point $x \in G$, or, dually, the value of the character $x \in G$ at the point $y \in G^{*}$. Let $\phi$ be a bounded complex Radon measure on $G$. The Fourier-Stieltjes transform of $\phi$ is defined as the function on $G^{*}$ equal to $\int_{G}(x, y) d \phi(x)$ for $y \in G^{*}$. It is shown that every bounded uniformly continuous complex function on $G^{*}$ can be represented as a Fourier-Stieltjes transform if and only if $G$ and $G^{*}$ are finite. This is accomplished by showing that if every continuous almost periodic function on $G^{*}$ is a Fourier-Stieltjes transform, then $G^{*}$ is finite. For a certain class of groups, it is shown that every continuous almost periodic function which is a Fourier-Stieltjes transform has an absolutely convergent Fourier series. (Received November 10, 1952.)

260. F. B. Jones: On certain well-ordered monotone collections of sets.

The study of Souslin spaces or nonmetric normal Moore spaces (such spaces are not known to exist) leads to certain situations analogous to the following: Suppose that $F$ is a well-ordered family of collections of mutually exclusive sets such that (1) if $F^{\prime}$ is a subsequence of $F$ which runs through $F$, then $\left|F^{\prime}\right|=|F|,(2)$ if $F^{\prime}$ is a subsequence of $F$ which does not run through $F$, then $\left|F^{\prime}\right|<|F|$, and (3) if $f$ is an element of $F$, each set in $f$ is a subset of some set in each preceding element of $F$. Theorem. If there exists a cardinal number $N$ such that for each element $f$ of $F,|f|<N<|F|$, then there exists a monotone collection $\alpha$ of sets such that each element of $F$ contains only one element of $\alpha$. (Received November 10, 1952.)

\section{W. S. Massey: Products in exact couples.}

Let $\langle A, C ; f, g, h\rangle$ be an exact couple of abelian groups (for the definitions and notation, see Ann. of Math. vol. 56 (1952) p. 367) which satisfies the following three conditions: (1) A multiplication is defined in $C$, making it a ring. (2) There are defined involutions $a \rightarrow \bar{a}$ and $c \rightarrow \bar{c}$ of $A$ and $C$ respectively such that $f(\bar{a})=(\overline{f a}), g(\bar{a})$ $=(\overline{g a})$, and $h(\bar{c})=-(\overline{h c})$. (3) For any integer $n \geqq 0$ and elements $a_{1}, a_{2} \in A, c_{1}, c_{2} \in C$ such that $h\left(c_{1}\right)=f^{n}\left(a_{1}\right), h\left(c_{2}\right)=f^{n}\left(a_{2}\right)$, there exists an element $a \in A$ such that $h\left(c_{1} c_{2}\right)$ $=f^{n}(a)$ and $g(a)=g\left(a_{1}\right) \cdot c_{2}+\bar{c}_{1} \cdot g\left(a_{2}\right)$ (here $f^{n}$ denotes the $n$th iterate of $f$ ). It is then possible to define a multiplication in $C^{\prime}$ and involutions of $A^{\prime}$ and $C^{\prime}$ such that these three conditions also hold for the derived exact couple $\left\langle A^{\prime}, C^{\prime} ; f^{\prime}, g^{\prime}, h^{\prime}\right\rangle$. The case 
is also considered where $A$ is a ring, $g$ is a ring homomorphism, and various conditions are imposed on $f$ and $h$. It is shown that the purely algebraic aspects of the theory of filtered rings with a differential operator (cf. J. Leray, J. de Math. vol. 29) may be subsumed under that of exact couples satisfying the above three conditions. (Received November 12, 1952.)

\section{E. A. Michael: An existence theorem for continuous functions.}

Theorem: The following condition is necessary and sufficient for a $T_{1}$-space to be paracompact (resp. normal): If $E$ is a Banach space (resp. separable Banach space), and $\tilde{f}$ a function from $X$ into the space of nonempty, closed (resp. compact), convex subsets of $E$ such that $\{x \in X \mid \tilde{f}(x) \cap U \neq \phi\}$ is open in $X$ for every open $U \subset E$, then there exists a continuous $f: X \rightarrow E$ such that $f(x) \in \tilde{f}(x)$ for every $x$ in $X$. Among the immediate consequences of this theorem, the author obtains the following known results. (1) (Katetov-Tong) For any $T_{1}$-space $X$, the following condition is equivalent to normality: If $R$ is the real line, $g: X \rightarrow R$ upper semi-continuous, $h: X \rightarrow R$ lower semi-continuous, and $g \leqq h$, then there exists a continuous $f: X \rightarrow R$ such that $g \leqq f \leqq h$. (2) (Arens) If $X$ is paracompact and $E$ a Banach space, then every continuous function from a closed subset of $X$ into $E$ can be extended to a continuous function from $X$ into $E$. (3) (Bartle-Graves) If $E$ is a Banach space, and $F$ a closed subspace of $E$, then there exists a continuous $f: E / F \rightarrow E$ such that $f(x) \in x$ for every $x$ in $X$. (Received November 6, 1952.)

\section{L. V. Toralballa: Local topological rings.}

Some algebraic and topological properties of a system $\Sigma$ with double composition satisfying the following conditions are considered: $1 . \Sigma$ is a topological space. $2 . \Sigma$ is a commutative local topological group with respect to addition. $3 . \Sigma$ is closed and associative with respect to multiplication. 4. Multiplication is continuous with respect to both factors. 5. If $b+c$ is defined, then so are $a+b+a \cdot c$ and $b \cdot a+c \cdot a$ and both distributive laws hold. 6 . There exists a neighborhood of zero which is homeomorphic to a neighborhood of the origin of a Euclidean space. (Received November 13, 1952.)

\section{H. C. Wang: Complex completely parallelisable manifolds.}

Let $M$ be a complex manifold of $2 n$ dimension. It is called complex completely parallelisable if there exist, over $M, n$ everywhere linearly independent complex analytic tangent vector fields. Unlike the real analytic case, such manifolds, if closed, cannot be simply-connected. It is shown that each compact, complex completely parallelisable manifold is analytically homeomorphic with the coset space $G / H$ of a complex Lie group over a discrete subgroup $H$ of $G$. Most of the complex Lie groups do not have any discrete subgroup $D$ such that $G / D$ is compact. The condition for $G$ to possess such a discrete subgroup is studied. (Received November 13,1952.)

\section{L. E. Ward, Jr.: Partially ordered spaces. I.}

Let $X$ be a partially ordered space, that is, a partially ordered set with topology in which the order relation, $\leqq$, is semi-continuous in the sense of Wallace [Bull. Amer. Math. Soc. vol. 51, pp. 413-416]. A subset $C$ of $X$ is a chain if it is linearly ordered with respect to $\leqq . X$ has closed bounded property (=CBP) if, whenever $A$ is a closed subset of $X, a \in X$, there are $q(a), p(a) \in A$ such that $q(a)$ is minimal in $A, p(a)$ is maximal in $A$, and $q(a) \leqq a \leqq p(a) . X$ has CBP if and only if every maximal chain 
of $X$ is compact. Thus every compact partially ordered space has CBP. By means of an argument employing monotone nets and a result of Nachbin, some fixed point theorems are proved for order-preserving maps $f: X \rightarrow X$, where $X$ has CBP. (Received November 12, 1952.)

\section{J. W. T. Youngs,} Associate Secretary

\section{APPENDIX}

\section{EXCERPTS FROM REPORT OF TREASURER}

To the Board of Trustees of the American Mathematical Society:

Gentlemen:

I have the honor to submit herewith the report of the Treasurer for the fiscal year ended November 30,1952, as is customary in two parts, (1) exhibits and schedules, and (2) a discussion and commentary.

On November 30,1952, the market value of securities held for invested funds exceeded book value by $\$ 27,767$, while the market value of securities held for current funds was less than book value by $\$ 440.00$.

During the year the book value of securities was revalued incident to the addition to the investment pool of $\$ 50,000$ derived from the sale of the Library. Hence the excess of market value over book value as of November 30, 1952 is not comparable with the figure given in last year's report. Our return this year on our invested funds has been 5.21 percent computed on average book value of investments.

Following is a summary of the changes in security holdings made during the year:

Acquired as result of stock splits

150 shares American Can Co.

3 shares Standard Oil Co. of New Jersey

During the year the accounts of the 1950 International Congress of Mathematicians were closed, and the sum of slightly over $\$ 20,000$ added to the Society's surplus. By action of the Board of Trustees, the funds accumulated as a reserve for possible reprinting of out-ofprint numbers of the Bulletin were transferred to surplus, since a committee appointed by the Council decided that it was unnecessary ever to reprint issues of the Bulletin. Similarly, the Transactions reprinting fund was reduced to $\$ 2,500$, with a transfer of $\$ 5,094.43$ 
to surplus, because the same committee felt that the Transactions need be kept in print only for ten years. In the judgment of the Trustees the sum of $\$ 2,500$ is an adequate reserve against the contingency of reprinting numbers which appeared so recently.

The Trustees have also adopted the policy of using annually the income of all non-restricted funds. Hitherto only the Endowment Fund income was thus utilized for the general purposes of the Society. Accumulated balances of something over $\$ 2,000$ were transferred to surplus and additional investment income of $\$ 4,000$ for the year used for general purposes.

It is a pleasure to report that the Society has lived within its income this year. Receipts for general purposes amounted to $\$ 93,000$; the general expenses of the Society were approximately $\$ 41,000$, the Bulletin, Proceedings and Transactions deficit amounted to just under $\$ 36,000$, to which should be added appropriations to other publications of some $\$ 6,500$, or a total for general publications of $\$ 42,000$. This left a surplus for the year of approximately $\$ 10,000$.

An additional schedule has been added to the exhibits, namely Exhibit $\mathrm{C}$ which shows the cost of operating the headquarters building. It is believed that members of the Society will be generally interested in the cost of maintaining our own headquarters. The cost of operating the building has been allocated to the various activities housed in it, namely the several publications, the Accounting Department and the Office of the Executive Director. Thus the building account does not explicitly appear on any of the other schedules.

It should perhaps also be pointed out that $\$ 3,500$ has been appropriated to repay the Library Proceeds Funds for the cost of moving to Providence and preparing the headquarters building for occupancy. The full cost of the relocation of the Society's office should have been recovered in five years.

While the financial problems of the Society continue to be a concern of the Board of Trustees, the return to a balanced budget for the year 1952 and, it is anticipated, for the year 1953, would indicate, in the absence of unforeseen contingencies, that for the next few years at least the Society can continue its activities on the present scale without further increase of dues. This statement, however, is contingent upon the continuation of Government support for Mathematical Reviews, and indeed its increase, for the Mathematical Reviews account incurred a deficit of $\$ 4,000$ this year.

Respectfully submitted, Albert E. Meder, JR. 


\section{BALANCE SHEET}

Assets

\begin{tabular}{|c|c|c|}
\hline \multicolumn{3}{|l|}{ Assets } \\
\hline & $\begin{array}{c}\text { November } 30, \\
1952\end{array}$ & $\begin{array}{c}\text { November } 30, \\
1951\end{array}$ \\
\hline \multicolumn{3}{|l|}{ CURRENT FUNDS: } \\
\hline \multirow{4}{*}{$\begin{array}{l}\text { Cash } \ldots \ldots \ldots \ldots \ldots \ldots \ldots \ldots \ldots \ldots \ldots \ldots \ldots \ldots \ldots \\
\text { Account Receivable (United States Government) } \ldots \\
\text { Investments } \ldots \ldots \ldots \ldots \ldots \ldots \ldots \ldots \ldots \ldots\end{array}$} & $\$ 19,430.02$ & $\$ 39,634.51$ \\
\hline & $4,667.27$ & $3,137.82$ \\
\hline & $67,329.96$ & $67,329.31$ \\
\hline & $\$ 91,427.25$ & $\$ 110,101.64$ \\
\hline \multicolumn{3}{|l|}{ INVESTED FUNDS: } \\
\hline \multirow{3}{*}{ 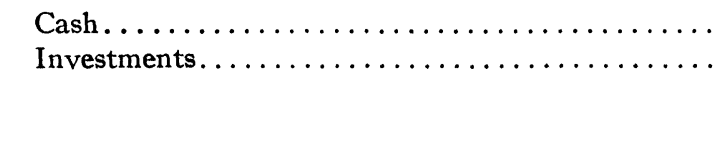 } & $\$ 1,109.03$ & $\$ 1,427.00$ \\
\hline & $288,090.07$ & $244,972.71$ \\
\hline & $\$ 289,199.10$ & $\$ 246,399.71$ \\
\hline 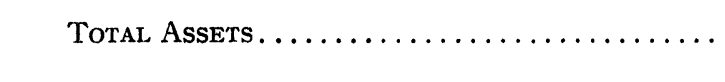 & $\$ 380,626.35$ & $\$ 356,501.35$ \\
\hline
\end{tabular}

\section{Liabilities}

Current Funds:

Publications.

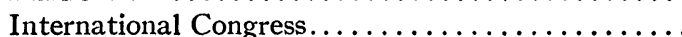

Policy Committee...........................

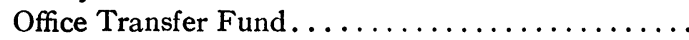

Combined List of Members..................

Prize Funds and Accumulated Income...........

Profit/Loss on Sales of Securities...............

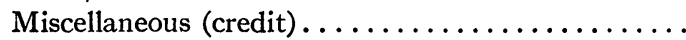

Account Payable (United States Government).....

Surplus (See Exhibit B)

Reserve for Proceedings and Transactions deficit (See Exhibit B)

$(\$ 5,042.85)$

$5,667.37$ $41,992.58$

$858.01 \quad 778.30$

$2,865.83 \quad 6,549.19$

( $4,685.55)$

$2,405.27 \quad 4,346.02$

$2,049.85 \quad 2,049.85$

$1,809.67 \quad 2,033.45$

28.95

$70,741.77 \quad 23,485.26$

$20,425.25 \quad 23,170.67$

$\$ 91,427.25 \$ 110,101.64$

\section{INVESTED FUNDS:}

Endowment Fund Principal. ...............

Library Proceeds Fund....................

Other Expendable Funds...................

Prize Funds. ..........................

Life Membership and Subscription Reserve.......

Mathematical Reviews.....................

Reserve for Investment Losses.................

Profit/Loss on Sales of Securities.

$$
\begin{array}{rrr}
\$ 94,000.00 & \$ 71,000.00 \\
50,000.00 & 50,000.00 \\
28,551.47 & 28,301.47 \\
6,575.00 & 5,381.75 \\
3,822.48 & 4,162.64 \\
80,000.00 & 65,000.00 \\
5,550.00 & 4,385.89 \\
20,700.15 & 18,167.96 \\
\cline { 1 - 1 } \$ 289,199.10 & & \$ 246,399.71 \\
\hline \$ 380,626.35 & & \$ 356,501.35
\end{array}
$$$$
\text { Total Liabilities }
$$ 


\section{GENERAL RECEIPTS AND DISBURSEMENTS}

GENERAL RECEIPTS:

Dues from Ordinary Memberships

$\$ 50,520.65$

Dues from Contributing Memberships.

702.53

Dues from Institutional Memberships. .

$21,995.17$

277.50

Publication Charges from Non-Member Institutions. .

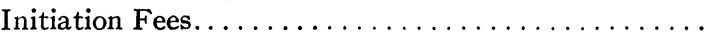

904.87

Income from

Henderson Estate. .

$\$ 7,300.00$

Current Funds Investments........ 1,508.34

Invested Funds............... $9,486.32$

$\$ 18,294.66$

Miscellaneous

Donations...................... . $\quad 34.95$

Meeting Fees. . . . . . . . . . . . . . . . . . . $\quad 669.80$

$\$ 704.75$

Total General Receipts

General Disbursements:

\section{General Activities}

Accounting Department.

Executive Director.

New Haven Office.

Officers' Expense .

Policy Committee

Theoretical Mechanics.

\section{Publications}

Bulletin.

American Journal.

Canadian Journal .

Pacific Journal. .

Annals...

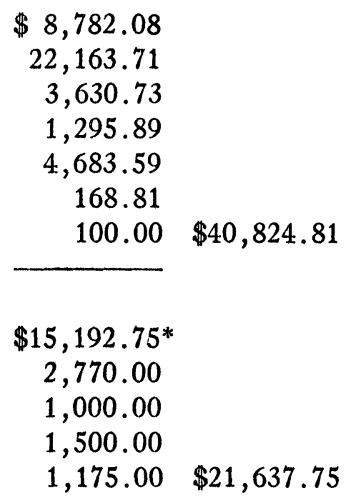

$1,295.89$

$4,683.59$

168.81

$100.00 \$ 40,824.81$

$\$ 15,192.75^{*}$

$2,770.00$

$1,000.00$

$1,500.00$

$1,175.00 \$ 21,637.75$

Total General Disbursements. . . . . . . . . . .

Excess of General Receipts over General Disbursements..... \$ $\$ 30,937.57$

* Receipts $\$ 3,223.19$, Disbursements $\$ 18,415.94$. 


\section{ANALYSIS OF SURPLUS}

Surplus at December 1, 1951

Additions Deductions

Adjustments to Previous Year

Excess of General Receipts over General Disbursements (see above).........

Bulletin Reprinting Fund............

Transactions Reprinting Fund.........

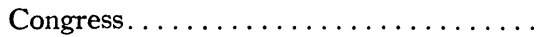

Transfer of Accumulated Balances in Expendable Funds.

Adjustments: Life Membership and Subscription Reserve, Library. $\$ 500.01 \$ 1,461.97$

$30,937.57$

$9,975.21$

$5,094.43$

$20,417.59$

$2,129.31$

$\frac{340.16}{\$ 69,394.28} \frac{250.55}{\$ 1,712.52}$

Net Additions

Surplus before Reserves

$\$ 91,167.02$

Reserves for Proceedings and Transactions Deficit...

$20,425.25$

SURPLUS, November 30,1952

EXHIBIT C

\section{COST OF OPERATING THE HEADQUARTERS BUILDING}

\section{BUILDING EXPENSE:}

Headquarters Utility Expense $\ldots \ldots \ldots \ldots \ldots \ldots \ldots \ldots \ldots \ldots$

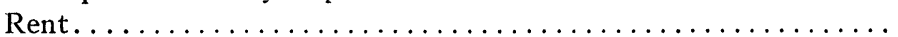

$\$ 1,371.68$

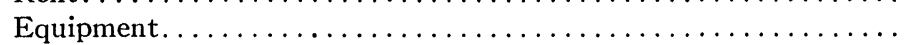
$2,400.00$

$1,374.59$

203.07

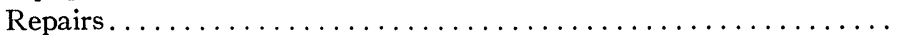

$1,226.51$

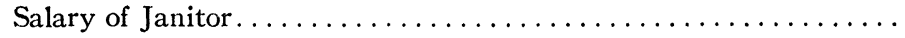

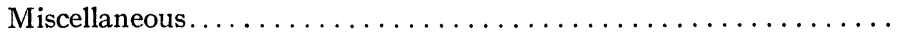

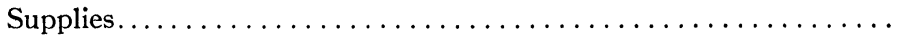

8.80

355.30

Amortization of Office Transfer................... 3,500.00

Total .................................. $\$ 10,439.95$

\section{EXHIBIT D}




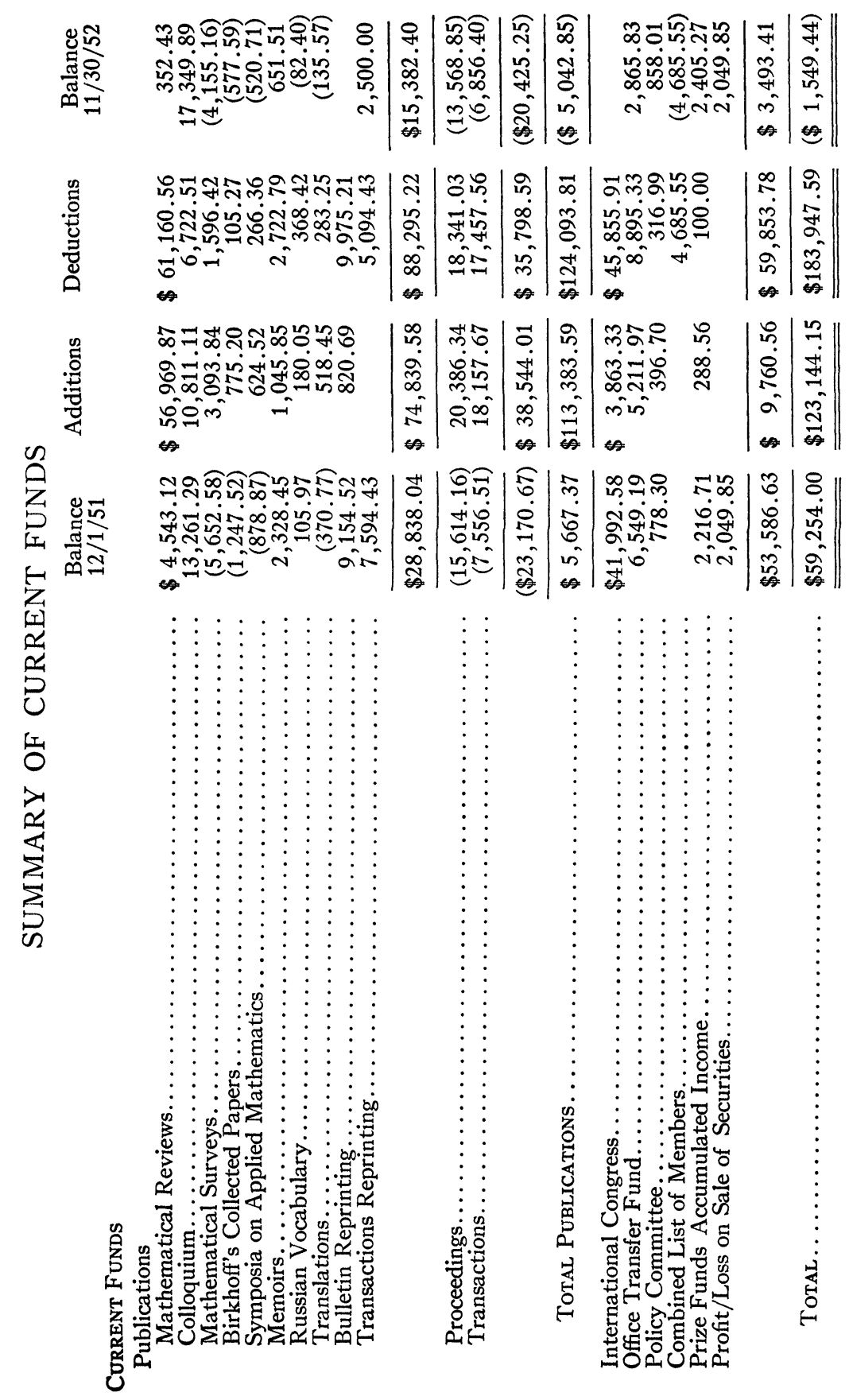




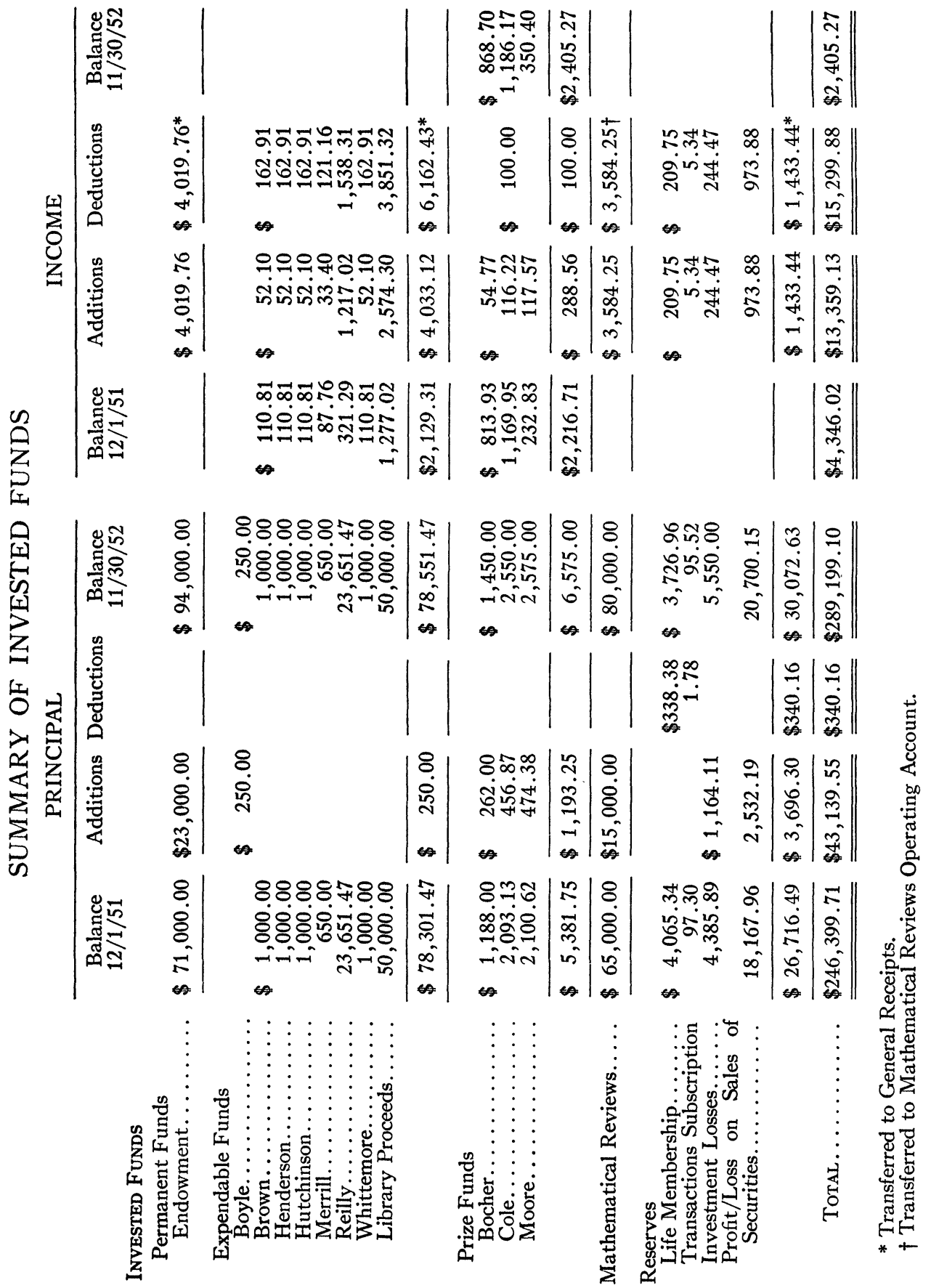

